Research Article

\title{
Synthesis, Characterization, and Antibacterial and Anti-Inflammatory Activities of New Pyrimidine and Thiophene Derivatives
}

\author{
Siham Lahsasni $\left(\mathbb{D},{ }^{1}\right.$ Dunya A. M. Al-Hemyari, ${ }^{1}$ Hazem A. Ghabbour $\left(\mathbb{D},{ }^{2,3}\right.$ \\ Yahia Nasser Mabkhoot $\mathbb{D}^{1}{ }^{1}$ Fadilah S. Aleanizy, ${ }^{1}$ Asma A. Alothman, ${ }^{1}$ \\ and Zainab M. Almarhoon ${ }^{1}$ \\ ${ }^{1}$ Department of Chemistry, King Saud University, P.O. Box 2455, Riyadh 11451, Saudi Arabia \\ ${ }^{2}$ Department of Pharmaceutical Chemistry, College of Pharmacy, King Saud University, Riyadh 11451, Saudi Arabia \\ ${ }^{3}$ Department of Medicinal Chemistry, Faculty of Pharmacy, University of Mansoura, Mansoura 35516, Egypt
}

Correspondence should be addressed to Siham Lahsasni; slahsasni@ksu.edu.sa

Received 17 May 2018; Revised 1 July 2018; Accepted 10 July 2018; Published 1 August 2018

Academic Editor: Gabriel Navarrete-Vazquez

Copyright (C) 2018 Siham Lahsasni et al. This is an open access article distributed under the Creative Commons Attribution License, which permits unrestricted use, distribution, and reproduction in any medium, provided the original work is properly cited.

\begin{abstract}
Substituted $[4,5]$ thieno[2,3- $d]$ thiazolo[3,2-a]pyrimidin-5-one (3a-b) and pyrimidin-5(6H)-imine $(3 \mathbf{c}-\mathbf{e})$ were synthesized via reaction of the starting compounds, ethyl 2-amino-substituted[b]thiophene-3-carboxylate (2a-c) and 2-amino-substituted [b]thiophene-3-carbonitrile (2d-f), respectively, with 2-bromothiazole. Synthesis of (bromo-substituted $[b]$ thiophen-2-yl) alkanamide derivatives (4a-e) and thieno[2,3-d][1,3]oxazin-4-imine derivative (5) was accomplished via reaction of the starting compounds with bromoalkyl chloride through nucleophilic substitution; however, for the synthesis of compound 5, nucleophilic substitution was followed by nucleophilic addition to a nitrile group to form the oxazinimine ring. 1-(3-cyanosubstituted $[b]$ thiophen-2-yl)-3-(4-(trifluoromethyl)phenyl)thiourea derivatives $(\mathbf{6 a}-\mathbf{c})$ were obtained via reaction of the starting compounds (2d-f) and 4-(trifluoromethyl phenyl)isothiocyanate. The lead compounds (2d-f) rapidly reacted with 4-(trifluoromethyl)benzaldehyde or 4-(2-pyridyl)benzaldehyde in acidic medium to yield compounds (7a-f) in large quantities. $\mathrm{X}$-ray crystallography of compounds $\mathbf{4 c}$ and $7 \mathbf{e}$ confirmed their structures. Antimicrobial studies revealed that compound $\mathbf{6 a}$ was equally potent to ampicillin against Bacillus strains. Moreover, compounds $\mathbf{3 e}, \mathbf{4 d}$, and $\mathbf{6 a}$ possessed greater anti-inflammatory potency than that of the standard drug.
\end{abstract}

\section{Introduction}

Thiophene, thiazole, and pyrimidine derivatives have been used as therapeutic drugs over years. Figure 1 shows some potent drugs containing thiazole or thiophene ring.

Dasatinib is a dual Src/Abl [1] and pan-Src [2] kinase inhibitor, zopolrestat [3] and lidorestat [4] are aldose reductase inhibitors used for the treatment of diabetic complications, and raloxifene is the first clinically available selective estrogen receptor modulator (SERM) used to prevent both osteoporosis and breast cancer [5-8]. In addition, clopidogrel is an antiplatelet agent used to inhibit blood clots in coronary artery disease and to prevent heart attacks and strokes in patients with heart or circulatory diseases [9-11].

The most efficient protocol for the synthesis of these thiophene derivatives is intramolecular cyclization via nucleophilic displacement [12-15], Gewald method [16, 17], thio-Claisen rearrangement [18], and dehydrophotocyclization $[19,20]$.

Within the scope of these diverse synthetic methods and utility of thiophene-based systems and in continuation to our interest in the design of bioactive heterocycles [21-23], we aimed at developing novel heterocyclic compounds containing thiophene, thiazole, and pyrimidine rings, identifying their structures using infrared (IR), ${ }^{1} \mathrm{H}$ nuclear 


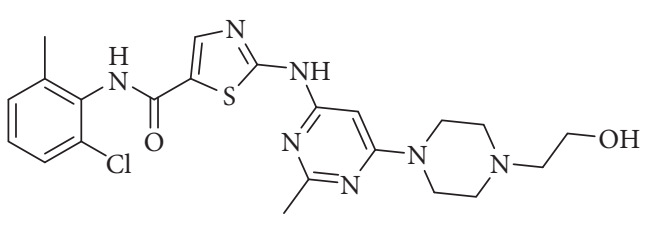

Dasatinib

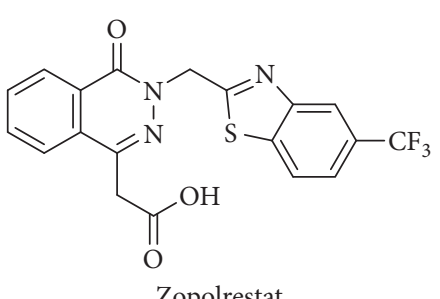

Zopolrestat<smiles>O=C(O)Cn1cc(Cc2nc3c(F)c(F)cc(F)c3s2)c2ccccc21</smiles>

Lidorestat

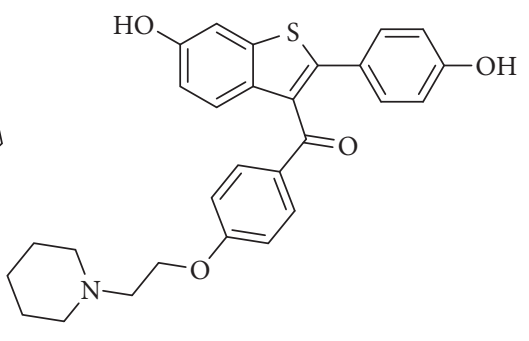

Raloxifene<smiles>COC(=O)[C@H](c1ccccc1Cl)N1CCc2sccc2C1</smiles>

Clopidogrel

FIGURE 1: Some drugs containing thiazole, pyrimidine, or thiophene ring.

magnetic resonance $\left({ }^{1} \mathrm{H} \mathrm{NMR}\right),{ }^{13} \mathrm{C}$ NMR spectroscopy, and $\mathrm{X}$-ray, and evaluating their biological effects, in particular, their antimicrobial and anti-inflammatory activities.

\section{Materials and Methods}

2.1. General Information. Melting points (mp) were determined using an electrothermal digital melting point apparatus and were uncorrected. IR spectra ( $\mathrm{KBr}$ discs) were recorded using FTIR plus 460 or Pye Unicam SP-1000 spectrophotometer. ${ }^{1} \mathrm{H}$ NMR spectra were recorded using Varian Gemini-200 (200 MHz) and Jeol AS (500 MHz) instruments. Dimethyl sulfoxide- (DMSO-) d6 was used as a solvent, and tetramethylsilane (TMS) was used as an internal standard. Chemical shifts were expressed as $\delta$ ppm. Mass spectra were recorded using Hewlett Packard 5988 A GC/MS system and GCMS-QP 1000 Ex Shimadzu instrument. Vario EL III Elemental CHNS analyzer at the Microanalytical Data Unit of Cairo University was used to obtain the analytical data.

\subsection{Chemistry}

2.2.1. Synthetic Procedure for Substituted [4,5]Thieno[2,3-d] thiazolo[3,2-a]pyrimidine Derivatives $(\mathbf{3 a}-\boldsymbol{e})$. A mixture of the appropriate lead compound (2b-f) $(5 \mathrm{mmol})$, an excess amount of 2-bromothiazole (approximately $1 \mathrm{~mL}$ ), and few drops of hydrochloric acid in absolute ethanol $(3 \mathrm{~mL})$ was refluxed for $3-5 \mathrm{~h}$. The product started to precipitate shortly once the reaction started (within approximately $15 \mathrm{~min}$ ). The precipitate was filtered, washed with absolute ethanol, dried, and recrystallized from absolute ethanol.

6,7,8,9-Tetrahydro-5H-benzo[4,5] thieno[2,3-d] thiazolo[3,2a]pyrimidin-5-one (3a). White solid; yield: 30\%; mp: $221-$ $222^{\circ} \mathrm{C}$; IR ( $\mathrm{KBr}, v_{\max }, \mathrm{cm}^{-1}$ ): 3079.1 ( $\mathrm{CH}$ aromatic), 2930.47 ( $\mathrm{CH}$ aliphatic), $1675.45(\mathrm{C}=\mathrm{O})$, and $1563.55,1516.11(\mathrm{C}=\mathrm{C}$ aromatic); ${ }^{1} \mathrm{H} \mathrm{NMR}\left(\delta, \mathrm{ppm}, \mathrm{CDCl}_{3}\right): 1.878(m, 4 \mathrm{H}, J=6 \mathrm{~Hz}$, H7-8), 2.77 ( $t, 2 \mathrm{H}, J=6 \mathrm{~Hz}, \mathrm{H} 9), 3.049(t, 2 \mathrm{H}, J=6 \mathrm{~Hz}, \mathrm{H} 6)$, $6.87(d, 1 \mathrm{H}, J=4.8 \mathrm{~Hz}, \mathrm{H} 2)$, and $7.98(d, 1 \mathrm{H}, J=4.8 \mathrm{~Hz}, \mathrm{H} 3) ;{ }^{13} \mathrm{C}$ $\operatorname{NMR}\left(\delta, \mathrm{ppm}, \mathrm{CDCl}_{3}\right): 22.253,22.95,25.158,25.618$ (C6-9), 109.571 (C2), 116.155 (C5a), 121.652 (C5a'), 130.973 (C3), 131.479 (C9a), 154.951 (C10a), 157.419 (C11a), and 163.835 (C5); and DART-TOF-MS $(\mathrm{m} / z): 263.03[\mathrm{M}+\mathrm{H}]^{+}$; anal. calcd. for $\mathrm{C}_{12} \mathrm{H}_{10} \mathrm{~N}_{2} \mathrm{OS}_{2}$ (262.02): C, 54.94; $\mathrm{H}, 3.84 ; \mathrm{N}, 10.68 ; \mathrm{O}, 6.10$; and S, 24.44; found: C, 55.04; H, 3.74; N, 10.60; O, 6.17; and S, 24.53 .

7,8,9,10-Tetrahydrocyclohepta[4,5] thieno [2,3-d] thiazolo [3,2-a]pyrimidin-5(6H)-one (3b). White solid; yield: 38\%; $\mathrm{mp}$ : $145-147^{\circ} \mathrm{C}$; IR $\left(\mathrm{KBr}, v_{\max }, \mathrm{cm}^{-1}\right): 3130.08(\mathrm{CH}$ aromatic), 2917.76 ( $\mathrm{CH}$ aliphatic), $1674.41(\mathrm{C}=\mathrm{O})$, and 1563.5, 1509.46 (C=C aromatic); ${ }^{1} \mathrm{H} \operatorname{NMR}\left(\delta, \mathrm{ppm}, \mathrm{CDCl}_{3}\right): 1.72$ ( $m, 4 \mathrm{H}, \mathrm{H} 7, \mathrm{H} 9), 1.897$ ( $m, 2 \mathrm{H}, \mathrm{H} 8), 2.838(t, 2 \mathrm{H}, J=5.4 \mathrm{~Hz}$, $\mathrm{H} 10), 3.365$ ( $t, 2 \mathrm{H}, J=4.8 \mathrm{~Hz}, \mathrm{H} 6), 6.855(d, 1 \mathrm{H}, J=4.8 \mathrm{~Hz}$, $\mathrm{H} 2)$, and $7.984(d, 1 \mathrm{H}, J=4.8 \mathrm{~Hz}, \mathrm{H} 3) ;{ }^{13} \mathrm{C} \operatorname{NMR}(\delta, \mathrm{ppm}$, $\left.\mathrm{CDCl}_{3}\right): 27.212,27.672,27.925,29.895,32.486$ (C6-10), 109.494 (C2), 116.853 (C5a), $121.667\left(\mathrm{C}^{\prime} \mathrm{a}^{\prime}\right), 135.634$ (C3), 136.438 (C10a), 155.403 (C11a), 156.982 (C12a), and 162.317 (C5); and DART-TOF-MS $(\mathrm{m} / z): 277.05[\mathrm{M}+\mathrm{H}]^{+}$; anal. calcd. for $\mathrm{C}_{13} \mathrm{H}_{12} \mathrm{~N}_{2} \mathrm{OS}_{2}$ (276.04): C, 56.50; $\mathrm{H}, 4.38 ; \mathrm{N}$, 10.14; O, 5.79; and S, 23.20; found: C, 56.43; H, 4.40; N, 10.18; O, 5.73; and S, 23.29.

7,8-Dihydrocyclopenta[4,5] thieno[2,3-d] thiazolo[3,2-a] pyrimidin-5(6H)-imine (3c). Dark green solid; yield: $22 \%$; $\mathrm{mp}<300^{\circ} \mathrm{C}$; IR $\left(\mathrm{KBr} v_{\max }, \mathrm{cm}^{-1}\right): 3241.38(\mathrm{NH}), 3073.4$ (CH aromatic), $1643.81(\mathrm{C}=\mathrm{N})$, and $1590.95,1509.8(\mathrm{C}=\mathrm{C}$ aromatic); ${ }^{1} \mathrm{H}$ NMR $\left(\delta\right.$, ppm, DMSO- $\left.d_{6}\right): 2.469(m, 2 \mathrm{H}, \mathrm{H} 7)$, $3.005(t, 2 \mathrm{H}, \mathrm{H} 8), 3.15(t, 2 \mathrm{H}, \mathrm{H} 6), 8.05(d, 1 \mathrm{H}, J=4.8 \mathrm{~Hz}, \mathrm{H} 2)$, $8.653(d, 1 \mathrm{H}, J=4.8 \mathrm{~Hz}, \mathrm{H} 3)$, and $9.172(s, 1 \mathrm{H}, \mathrm{NH})$; and ${ }^{13} \mathrm{C}$ $\operatorname{NMR}\left(\delta\right.$, ppm, DMSO- $\left.d_{6}\right): 27.664,29.496,29.971$ (C6-8), 108.682 (C2), 118.210 (C5a), 122.395 (C5a'), 136.416 (C8a), 141.138 (C9a), 148.359 (C3), 157.121 (C5), and 168.972 (10a); and DART-TOF-MS $(\mathrm{m} / z): 248.03[\mathrm{M}+\mathrm{H}]^{+}$; anal. calcd. for $\mathrm{C}_{11} \mathrm{H}_{9} \mathrm{~N}_{3} \mathrm{~S}_{2}$ (247.02): C, 53.42; H, 3.67; N, 16.99; and S, 25.92; found: C, 53.47; H, 3.64; N, 17.05; and S, 25.89 .

6,7,8,9-Tetrahydro-5H-benzo[4,5] thieno[2,3-d] thiazolo [3,2-a]pyrimidin-5-imine (3d). Yellow solid; yield: $88 \%$; 
$\mathrm{mp}<300^{\circ} \mathrm{C}$; IR $\left(\mathrm{KBr}, v_{\max }, \mathrm{cm}^{-1}\right): 3281.42(\mathrm{NH}), 3080.16$ (CH aromatic), 2929.63 ( $\mathrm{CH}$ aliphatic), $1643.47(\mathrm{C}=\mathrm{N})$, and $1564.15,1504.9$ (C=C aromatic); ${ }^{1} \mathrm{H}$ NMR $(\delta$, ppm, DMSO$\left.d_{6}\right): 1.835(s, 4 \mathrm{H}, \mathrm{H} 7, \mathrm{H} 8), 2.824(s, 2 \mathrm{H}, \mathrm{H} 6), 2.993(s, 2 \mathrm{H}$, H9), $8.002(d, 1 \mathrm{H}, J=4.8 \mathrm{~Hz}, \mathrm{H} 2), 8.619(d, 1 \mathrm{H}, J=4.8 \mathrm{~Hz}$, $\mathrm{H} 3)$, and $9.002(s, 1 \mathrm{H}, \mathrm{NH}) ;{ }^{13} \mathrm{C}$ NMR $\left(\delta\right.$, ppm, DMSO- $\left.d_{6}\right)$ : 21.945, 22.144, 25.471, 25.931 (C6-9), 111.372 (C2), 118.003 (C5a), $122.319\left(\mathrm{C}^{\prime} \mathrm{a}^{\prime}\right), 128.091$ (C9a), 135.664 (C10a), 148.596 (C3), 157.573 (C5), and 164.303 (C11a); and DARTTOF-MS $(\mathrm{m} / z): 262.05[\mathrm{M}+\mathrm{H}]^{+}$; anal. calcd. for $\mathrm{C}_{12} \mathrm{H}_{11} \mathrm{~N}_{3} \mathrm{~S}_{2}$ (261.04): C, 55.15; H, 4.24; N, 16.08; and S, 24.53; found: C, 55.09; H, 4.31; N, 16.13; and S, 24.57 .

7,8,9,10-Tetrahydrocyclohepta[4,5] thieno[2,3-d] thiazolo [3,2-a]pyrimidin-5(6H)-imine (3e). Light brown solid; yield: $70 \%$; $\mathrm{mp}<300^{\circ} \mathrm{C}$; IR $\left(\mathrm{KBr}, v_{\max }, \mathrm{cm}^{-1}\right): 3270.42(\mathrm{NH})$, 3053.25 (CH aromatic), 2906.04 (CH aliphatic), 1639.62 $(\mathrm{C}=\mathrm{N})$, and $1556.71,1501.3\left(\mathrm{C}=\mathrm{C}\right.$ aromatic); ${ }^{1} \mathrm{H}$ NMR $(\delta$, ppm, DMSO- $\left.d_{6}\right): 1.714,1.866,2.953,3.12(m, 10 \mathrm{H}, \mathrm{H} 6-10)$, $8.02(d, 1 \mathrm{H}, \mathrm{H} 2), 8.63(d, 1 \mathrm{H}, \mathrm{H} 3)$, and $9.186(s, 1 \mathrm{H}, \mathrm{NH}) ;{ }^{13} \mathrm{C}$ $\operatorname{NMR}\left(\delta\right.$, ppm, DMSO- $\left.d_{6}\right): 26.485,26.914,28.838,28.93,30.67$ (C6-10), 112.192 (C2), 117.865 (C5a), 122.434 (C5a'), 133.319 (C10a), 139.765 (C11a), 148.941 (C3), 157.204 (C5), and 163.107 (C12a); and DART-TOF-MS ( $\mathrm{m} / z): 276.07[\mathrm{M}+\mathrm{H}]^{+}$; anal. calcd. for $\mathrm{C}_{13} \mathrm{H}_{13} \mathrm{~N}_{3} \mathrm{~S}_{2}$ (275.06): C, 56.70; H, 4.76; N, 15.26; and S, 23.28; found: C, 56.74; H, 4.72; N, 15.34; and S, 23.33 .

2.2.2. Synthetic Procedure for Compounds (4a-e, 5). Compounds $2 \mathbf{b}-\mathbf{f}(1.7 \mathrm{mmol})$ were refluxed in bromoalkanoyl chloride for $2-3 \mathrm{~h}$. Then, the mixture was left at room temperature until the product precipitated. The precipitate was filtered, washed with absolute ethanol, dried, and recrystallized from a mixture of absolute ethanol and methanol.

Ethyl-2-(5-bromopentanamido)-5,6-dihydro-4H-cyclopenta [b]thiophene-3-carboxylate (4a). Greenish white solid; yield: 25\%; mp: $57-59^{\circ} \mathrm{C}$; IR $\left(\mathrm{KBr}, v_{\max }, \mathrm{cm}^{-1}\right)$ : $3271.77(\mathrm{NH})$, 2944.81 ( $\mathrm{CH}$ aliphatic), $1669.36(\mathrm{C}=\mathrm{O})$, and $1558.46,1520.71$ $\left(\mathrm{C}=\mathrm{C}\right.$ aromatic); ${ }^{1} \mathrm{H}$ NMR $\left(\delta, \mathrm{ppm}, \mathrm{CDCl}_{3}\right): 1.353(t$, $\left.3 \mathrm{H}, J=6.6 \mathrm{~Hz},-\mathrm{CH}_{2} \mathrm{CH}_{3}\right), 1.916\left(m, 4 \mathrm{H}, \mathrm{H}^{\prime}-4^{\prime}\right), 2.356(m$, $2 \mathrm{H}, J=6.6 \mathrm{~Hz}, \mathrm{H} 5), 2.491\left(t, 2 \mathrm{H}, J=6.6 \mathrm{~Hz}, \mathrm{H} 2^{\prime}\right), 2.82$ $(t, 2 \mathrm{H}, \mathrm{H} 6), 2.867(t, 2 \mathrm{H}, \mathrm{H} 4), 3.419\left(t, 2 \mathrm{H}, J=5.4 \mathrm{~Hz}, \mathrm{H}^{\prime}\right)$, $4.29\left(q, 2 \mathrm{H}, J=6.6 \mathrm{~Hz},-\mathrm{CH}_{2} \mathrm{CH}_{3}\right)$, and $11.004(s, 1 \mathrm{H}, \mathrm{NH})$; ${ }^{13} \mathrm{C}$ NMR $\left(\delta, \mathrm{ppm}, \mathrm{CDCl}_{3}\right): 14.271\left(-\mathrm{CH}_{2} \mathrm{CH}_{3}\right), 22.481$, 23.731, 27.908, 28.828, 30.262, 31.940, 32.929, 35.589 (C4-6, $\left.\mathrm{C} 2^{\prime}-5^{\prime}\right), 60.441\left(-\mathrm{CH}_{2} \mathrm{CH}_{3}\right), 108.083(\mathrm{C} 3), 132.169$ (C6a), 141.214 (C3a), 151.341 ( $\mathrm{C}=\mathrm{O}$ ester), 166.143 ( $\mathrm{C}=\mathrm{O}$ amide), and $169.11(\mathrm{C} 2)$; and DART-TOF-MS $(\mathrm{m} / \mathrm{z}): 374.04[\mathrm{M}+$ $\mathrm{H}]^{+}$; anal. calcd. for $\mathrm{C}_{15} \mathrm{H}_{20} \mathrm{BrNO}_{3} \mathrm{~S}$ (373.03): C, 48.13; $\mathrm{H}$, 5.39; $\mathrm{Br}, 21.35 ; \mathrm{N}, 3.74 ; \mathrm{O}, 12.82$; and $\mathrm{S}, 8.57$; found: $\mathrm{C}$, 48.18; H, 5.24; Br, 21.41; N, 3.69; O, 12.77; and S, 8.52.

5-Bromo-N-(3-cyano-4,5,6,7-tetrahydrobenzo[b] thiophen-2-yl)pentanamide (4b). White solid; yield: $74 \%$; mp: $149-150^{\circ} \mathrm{C}$; IR (KBr, $\left.v_{\max }, \mathrm{cm}^{-1}\right): 3468.26(\mathrm{NH}), 2935.12$ ( $\mathrm{CH}$ aliphatic), $2214.8(\mathrm{CN}), 1699.82(\mathrm{C}=\mathrm{O})$, and 1548.87, $1455.74\left(\mathrm{C}=\mathrm{C}\right.$ aromatic); ${ }^{1} \mathrm{H}$ NMR $\left(\delta, \mathrm{ppm}, \mathrm{CDCl}_{3}\right)$ : 1.818-1.967 ( $\left.m, 8 \mathrm{H}, \mathrm{H} 5-6, \mathrm{H}^{\prime}-4^{\prime}\right) 2.569,2.614(m, 4 \mathrm{H}, \mathrm{H} 4$, $\mathrm{H} 7), 2.533\left(t, 2 \mathrm{H}, \mathrm{H} 2^{\prime}\right), 3.433\left(t, 2 \mathrm{H}, \mathrm{H} 5^{\prime}\right)$, and $9.277(s, 1 \mathrm{H}$,
$\mathrm{NH}) ;{ }^{13} \mathrm{C} \mathrm{NMR}\left(\delta, \mathrm{ppm}, \mathrm{CDCl}_{3}\right): 22.115,23.065,23.74$, 23.885, 23.954, 31.942, 32.946, $34.794\left(\mathrm{C} 4-7, \mathrm{C}^{\prime}{ }^{\prime}-5^{\prime}\right), 92.316$ (C3), 114.814 (CN), 128.083 (C7a), 130.689 (C3a), 147.507 (C2), and 169.829 (C=O amide); and DART-TOF-MS $(\mathrm{m} / z)$ : $341.03[\mathrm{M}+\mathrm{H}]^{+}$; anal. calcd. for $\mathrm{C}_{14} \mathrm{H}_{17} \mathrm{BrN}_{2} \mathrm{OS}$ (340.02): C, 49.27; H, 5.02; Br, 23.41; N, 8.21; O, 4.69; and S, 9.39; found: C, 49.32; H, 4.98; Br, 23.49; N, 8.17; O, 4.73; and S, 9.31.

5-Bromo-N-(3-cyano-5,6,7,8-tetrahydro-4H-cyclohepta[b] thiophen-2-yl)pentanamide (4c). Beige solid; yield: $82 \%$; mp: 127-129 C; IR (KBr, $\left.v_{\max }, \mathrm{cm}^{-1}\right): 3486.48(\mathrm{NH}), 2924.92$ ( $\mathrm{CH}$ aliphatic), $2213.55(\mathrm{CN}), 1696.13(\mathrm{C}=\mathrm{O})$, and 1544.89, 1441.07 (C=C aromatic); ${ }^{1} \mathrm{H} \operatorname{NMR}\left(\delta, \mathrm{ppm}, \mathrm{CDCl}_{3}\right): 1.656$ ( $m, 4 \mathrm{H}, \mathrm{H} 5, \mathrm{H} 7), 1.85-1.951\left(m, \mathrm{H}^{\prime}-4^{\prime}\right), 2.507(m, 2 \mathrm{H}, \mathrm{H} 6)$, $2.697(s, 4 \mathrm{H}, \mathrm{H} 4, \mathrm{H} 8), 3.431,3.567\left(m, 2 \mathrm{H}, \mathrm{H} 5^{\prime}\right)$, and 8.575 $(s, 1 \mathrm{H}, \mathrm{NH}) ;{ }^{13} \mathrm{C} \mathrm{NMR}\left(\delta, \mathrm{ppm}, \mathrm{CDCl}_{3}\right): 22.483,23.725$, 27.32, 27.994, 29.044, 31.758, 31.888, 32.003, 34.947 (C4-8, C2'-5'), 95.106 (C3), 115.159 (CN), 131.77 (C8a), 135.465 (C3a), 145.031 (C2), and 169.623 ( $\mathrm{C}=\mathrm{O}$ amide); and DART-TOF-MS $(\mathrm{m} / z)$ : $355.05[\mathrm{M}+\mathrm{H}]^{+}$; anal. calcd. for $\mathrm{C}_{15} \mathrm{H}_{19} \mathrm{BrN}_{2} \mathrm{OS}$ (354.04): C, 50.71; H, 5.39; Br, 22.49; N, 7.88; O, 4.50; and S, 9.02; found: C, 50.78; H, 5.34; Br, 22.41; N, 7.93; O, 4.47; and S, 8.96.

Ethyl-2-(6-bromohexanamido)-5,6,7,8-tetrahydro-4Hcyclohepta[b]thiophene-3-carboxylate (4d). White solid; yield: $43 \%$; $\mathrm{mp}$ : $66-67^{\circ} \mathrm{C}$; IR ( $\left.\mathrm{KBr}, v_{\max }, \mathrm{cm}^{-1}\right): 3360.95$ (NH), 2915.27 ( $\mathrm{CH}$ aliphatic), $1675.80(\mathrm{C}=\mathrm{O})$, and 1529.22 (C=C aromatic); ${ }^{1} \mathrm{H}$ NMR $\left(\delta, \mathrm{ppm}, \mathrm{CDCl}_{3}\right): 1.379(t, 3 \mathrm{H}$, $\left.J=7.2 \mathrm{~Hz},-\mathrm{CH}_{2} \mathrm{CH}_{3}\right), 1.508\left(m, 2 \mathrm{H}, \mathrm{H}^{\prime}\right), 1.594,1.638$ (4H, H5, H7), $1.756\left(m, 2 \mathrm{H}, \mathrm{H}^{\prime}\right), 1.826(s, 2 \mathrm{H}, \mathrm{H} 6), 1.888$ $\left(m, 2 \mathrm{H}, \mathrm{H} 5^{\prime}\right), 2.456\left(t, 2 \mathrm{H}, J=7.2 \mathrm{~Hz}, \mathrm{H} 2^{\prime}\right), 2.699(m, 2 \mathrm{H}$, H8), 3.015 ( $m, 2 \mathrm{H}, \mathrm{H} 4), 3.396\left(t, 2 \mathrm{H}, J=6.6 \mathrm{~Hz}, \mathrm{H}^{\prime}\right), 4.33$ $\left(q, 2 \mathrm{H}, J=7.2 \mathrm{~Hz},-\mathrm{CH}_{2} \mathrm{CH}_{3}\right)$, and $11.2(s, 1 \mathrm{H}, \mathrm{NH}) ;{ }^{13} \mathrm{C}$ $\operatorname{NMR}\left(\delta, \mathrm{ppm}, \mathrm{CDCl}_{3}\right): 14.25\left(-\mathrm{CH}_{2} \mathrm{CH}_{3}\right), 24.442,26.936$, 27.688, 27.795, 28.247, 28.561, 32.21, 32.417, 33.444, 36.564 (C4-8, C2'-6'), $60.649\left(-\mathrm{CH}_{2} \mathrm{CH}_{3}\right), 112.668$ (C3), 130.843 (C8a), 136.270 (C3a), 145.484 ( $\mathrm{C}=\mathrm{O}$ ester), $166.748(\mathrm{C}=\mathrm{O}$ amide), and 169.554 (C2); and DART-TOF-MS $(\mathrm{m} / z)$ : $416.09[\mathrm{M}+\mathrm{H}]^{+}$; anal. calcd. for $\mathrm{C}_{18} \mathrm{H}_{26} \mathrm{BrNO}_{3} \mathrm{~S}$ (415.08): C, 51.92; H, 6.29; $\mathrm{Br}, 19.19 ; \mathrm{N}, 3.36 ; \mathrm{O}, 11.53$; and $\mathrm{S}, 7.70$; found: C, 51.88; H, 6.34; Br, 19.25; N, 3.31; O, 11.47; and S, 7.79.

6-Bromo-N-(3-cyano-5,6,7,8-tetrahydro-4H-cyclohepta[b] thiophen-2-yl)hexan-amide( $4 \boldsymbol{e})$. Brown solid; yield: 69\%; mp: $104-106^{\circ} \mathrm{C}$; IR $\left(\mathrm{KBr}, v_{\max }, \mathrm{cm}^{-1}\right): 3420.51(\mathrm{NH}), 2925.32$ ( $\mathrm{CH}$ aliphatic), $2217.32(\mathrm{CN}), 1688.82(\mathrm{C}=\mathrm{O})$, and 1552.66, $1439.5\left(\mathrm{C}=\mathrm{C}\right.$ aromatic); ${ }^{1} \mathrm{H}$ NMR $\left(\delta, \mathrm{ppm}_{,} \mathrm{CDCl}_{3}\right): 1.529$ $\left(m, 2 \mathrm{H}, \mathrm{H} 4^{\prime}\right), 1.66(m, 4 \mathrm{H}, \mathrm{H} 5, \mathrm{H} 7), 1.765\left(m, 2 \mathrm{H}, \mathrm{H} 3^{\prime}\right), 1.85$ $(m, 2 \mathrm{H}, \mathrm{H} 6), 1.899\left(m, 2 \mathrm{H}, \mathrm{H}^{\prime}\right), 2.496(t, 2 \mathrm{H}, J=7.2 \mathrm{~Hz}$, $\left.\mathrm{H} 2^{\prime}\right), 2.694(m, 4 \mathrm{H}, \mathrm{H} 4, \mathrm{H} 8), 3.409\left(t, 2 \mathrm{H}, J=6.6 \mathrm{~Hz}, \mathrm{H}^{\prime}\right)$, and $8.91(s, 1 \mathrm{H}, \mathrm{NH}) ;{ }^{13} \mathrm{C} \mathrm{NMR}\left(\delta, \mathrm{ppm}, \mathrm{CDCl}_{3}\right): 24.322$, 27.327, 27.665, 27.994, 29.044, 29.067, 31.996, 32.364, 33.406, 35.706 (C4-8, C2'-6'), $95.075(\mathrm{C} 3), 115.151(\mathrm{CN}), 131.716$ (C8a), 135.411 (C3a), 145.07 (C2), and $169.921(\mathrm{C}=\mathrm{O}$ amide); and DART-TOF-MS $(\mathrm{m} / z)$ : $369.07[\mathrm{M}+\mathrm{H}]^{+}$; anal. calcd. for $\mathrm{C}_{16} \mathrm{H}_{21} \mathrm{BrN}_{2} \mathrm{OS}$ (368.06): C, 52.03; $\mathrm{H}, 5.73 ; \mathrm{Br}$, 21.64; N, 7.59; O, 4.33; and S, 8.68; found: C, 52.08f H, 5.64; Br, 21.69; N, 7.51; O, 4.37; and S, 8.62.

2-(5-Bromopentyl)-5,6,7,8-tetrahydro-4H-benzo[4,5]thieno [2,3-d][1,3]oxazin-4-imine (5). White solid; yield: 40\%; $\mathrm{mp}: 174-175^{\circ} \mathrm{C}$; IR $\left(\mathrm{KBr}, v_{\max }, \mathrm{cm}^{-1}\right): 2936.82(\mathrm{CH}$ 
aliphatic), $1664.9(\mathrm{C}=\mathrm{N})$, and $1588.12(\mathrm{C}=\mathrm{C}) ;{ }^{1} \mathrm{H}$ NMR $\left(\delta, \mathrm{ppm}, \mathrm{DMSO}-d_{6}\right): 1.376\left(m, 2 \mathrm{H}, \mathrm{H}^{\prime}\right), 1.641-1.816(m$, $\left.8 \mathrm{H}, \mathrm{H} 2^{\prime}, \mathrm{H} 4^{\prime}, \mathrm{H} 7-8\right), 2.553\left(t, 2 \mathrm{H}, J=6.6 \mathrm{~Hz}, \mathrm{H1}{ }^{\prime}\right), 2.686(s$, $2 \mathrm{H}, \mathrm{H} 6), 2.821(s, 2 \mathrm{H}, \mathrm{H} 6), 3.508(t, 2 \mathrm{H}, J=6.6 \mathrm{~Hz}, \mathrm{H} 5)$, and $12.177(s, 1 \mathrm{H}, \mathrm{NH}) ;{ }^{13} \mathrm{C}$ NMR $\left(\delta, \mathrm{ppm}, \mathrm{DMSO}-d_{6}\right)$ : $22.246,22.966,24.821,25.726,26.4,27.343,32.264$, 34.058, 35.469 (C6-9, C1'-5'), 120.778 (C4a), 130.965 (C4a'), 131.395 (C9a), 157.787 (C10a), 159.013 (C4), and 163.559 (C2); and DART-TOF-MS $(\mathrm{m} / z): 355.05[\mathrm{M}+\mathrm{H}]$ ${ }^{+}$; anal. calcd. for $\mathrm{C}_{15} \mathrm{H}_{19} \mathrm{BrN}_{2} \mathrm{OS}$ (354.04): C, 50.71; $\mathrm{H}$, 5.39; $\mathrm{Br}, 22.49 ; \mathrm{N}, 7.88 ; \mathrm{O}, 4.50$; and $\mathrm{S}, 9.02$; found: $\mathrm{C}$, 50.76; H, 5.32; Br, 22.52; N, 7.85; O, 4.53; and S, 8.96.

2.2.3. General Synthetic Procedure for 1-(3-Cyano-substituted [b]thiophen-2-yl)-3-(4-(trifluoromethyl)phenyl)thiourea (6a-c). A mixture of the appropriate compound $2 \mathbf{d}-\mathbf{f}(1.3 \mathrm{mmol})$ with 1 equivalent of 4-(trifluoromethyl)phenyl isothiocyanate in absolute ethanol was stirred at room temperature for $2-7 \mathrm{~h}$. The precipitate was filtered, washed with absolute ethanol, and dried.

1-(3-Cyano-5,6-dihydro-4H-cyclopenta[b]thiophen-2-yl)-3(4-(trifluoromethyl)phenyl)thiourea (6a). Light brown solid; yield: $31 \%$; $\mathrm{mp}$ : $172-174^{\circ} \mathrm{C}$; IR $\left(\mathrm{KBr}, v_{\max }, \mathrm{cm}^{-1}\right)$ : 3327.76 , $3234.11(\mathrm{NH}), 2212.49(\mathrm{CN})$, and $1589.8,1560.47(\mathrm{C}=\mathrm{C}) ;{ }^{1} \mathrm{H}$ $\operatorname{NMR}\left(\delta, \mathrm{ppm}, \mathrm{DMSO}-d_{6}\right): 2.323(s, 2 \mathrm{H}, \mathrm{H} 5), 2.72(s, 2 \mathrm{H}$, $\mathrm{H} 6), 2.814(s, 2 \mathrm{H}, \mathrm{H} 4), 7.701\left(s, 2 \mathrm{H}, \mathrm{H} 2^{\prime}, \mathrm{H}^{\prime}\right), 7.818(s, 2 \mathrm{H}$, $\left.\mathrm{H}^{\prime}, \mathrm{H}^{\prime}\right), 10.71(s, 1 \mathrm{H}, \mathrm{NH})$, and $10.95(s, 1 \mathrm{H}, \mathrm{NH}) ;{ }^{13} \mathrm{C}$ NMR $\left(\delta, \mathrm{ppm}, \mathrm{DMSO}-d_{6}\right): 27.786,28.039,29.649$ (C4-5), $115.036(\mathrm{CN}), 123.139\left(\mathrm{C}^{\prime}, \mathrm{C}^{\prime}\right), 126.236\left(\mathrm{C}^{\prime}, \mathrm{C}^{\prime}\right)$, and 130.613 (C3a); and DART-TOF-MS $(\mathrm{m} / \mathrm{z}): 368.05[\mathrm{M}+\mathrm{H}]^{+}$; anal. calcd. for $\mathrm{C}_{16} \mathrm{H}_{12} \mathrm{~F}_{3} \mathrm{~N}_{3} \mathrm{~S}_{2}$ (367.04): C, 52.31; H, 3.29; F, 15.51; N, 11.44; and S, 17.45; found: C, 52.27; H, 3.35; F, 15.55; N, 11.38; and S, 17.40.

1-(3-Cyano-4,5,6,7-tetrahydrobenzo[b]thiophen-2-yl)-3(4-(trifluoromethyl)phenyl)thiourea (6b). Light beige solid; yield: $49 \%$; $\mathrm{mp}$ : $187-189^{\circ} \mathrm{C}$; IR $\left(\mathrm{KBr}, v_{\max }, \mathrm{cm}^{-1}\right)$ : 3328.78 , $3235.86(\mathrm{NH}), 2210.19(\mathrm{CN})$, and 1590.92, $1560.13(\mathrm{C}=\mathrm{C})$; ${ }^{1} \mathrm{H}$ NMR $\left(\delta, \mathrm{ppm}, \mathrm{DMSO}-d_{6}\right): 1.74(s, 4 \mathrm{H}, \mathrm{H} 5-6), 2.498$ $(m, 2 \mathrm{H}, \mathrm{H} 7), 2.585(s, 2 \mathrm{H}, \mathrm{H} 4), 7.715(d, 2 \mathrm{H}, J=7.8 \mathrm{~Hz}$, $\left.\mathrm{H} 2^{\prime}, \mathrm{H}^{\prime}\right), 7.838\left(d, 2 \mathrm{H}, J=7.8 \mathrm{~Hz}, \mathrm{H}^{\prime}, \mathrm{H}^{\prime}\right), 10.765(s$, $1 \mathrm{H}, \mathrm{NH})$, and $11.016(s, 1 \mathrm{H}, \mathrm{NH}) ;{ }^{13} \mathrm{C} \operatorname{NMR}(\delta, \mathrm{ppm}$, DMSO- $\left.d_{6}\right): 22.146,23.05,23.809,23.848$ (C4-7), 96.041 (C3), $114.791(\mathrm{CN}), 123.07\left(\mathrm{C}^{\prime}, \mathrm{C}^{\prime}\right), 123.775\left(\mathrm{CF}_{3}\right)$, $125.584\left(\mathrm{C}^{\prime}\right), 126.297\left(\mathrm{C}^{\prime}, \mathrm{C}^{\prime}\right), 128.581$ (C3a), 130.988 (C7a), $142.862\left(\mathrm{Cl}^{\prime}\right), 148.895(\mathrm{C} 2)$, and $176.728(\mathrm{C}=\mathrm{S})$; and DART-TOF-MS $(\mathrm{m} / z): 382.07[\mathrm{M}+\mathrm{H}]^{+}$anal. calcd. for $\mathrm{C}_{17} \mathrm{H}_{14} \mathrm{~F}_{3} \mathrm{~N}_{3} \mathrm{~S}_{2}$ (381.06): C, 53.53; $\mathrm{H}, 3.70 ; \mathrm{F}, 14.94 ; \mathrm{N}$, 11.02; and S, 16.81; found: C, 53.57; H, 3.64; F, 15.0; N, 11.06; and S, 16.75 .

1-(3-Cyano-5,6,7,8-tetrahydro-4H-cyclohepta[b]thiophen2-yl)-3-(4-(trifluoro-methyl)phenyl)thiourea (6c). Dark beige solid; yield: $32 \%$; $\mathrm{mp}$ : $169-171^{\circ} \mathrm{C}$; IR $\left(\mathrm{KBr}, v_{\max }, \mathrm{cm}^{-1}\right)$ : $3326.54(\mathrm{NH}), 2211.97(\mathrm{CN})$, and 1582.92, $1554.87(\mathrm{C}=\mathrm{C})$; ${ }^{1} \mathrm{H}$ NMR $\left(\delta\right.$, ppm, DMSO- $\left.d_{6}\right): 1.572(s, 4 \mathrm{H}, \mathrm{H} 5, \mathrm{H} 7), 1.781$ $(s, 2 \mathrm{H}, \mathrm{H} 6), 2.633-2.674(m, 4 \mathrm{H}, \mathrm{H} 4, \mathrm{H} 8), 7.705(d, 2 \mathrm{H}$, $\left.J=8.4 \mathrm{~Hz}, \mathrm{H}^{\prime}, \mathrm{H} 6^{\prime}\right), 7.828\left(d, 2 \mathrm{H}, J=8.4 \mathrm{~Hz}, \mathrm{H}^{\prime}{ }^{\prime}, \mathrm{H}^{\prime}\right)$, $10.777(s, 1 \mathrm{H}, \mathrm{NH})$, and $10.937(s, 1 \mathrm{H}, \mathrm{NH}),{ }^{13} \mathrm{C} \mathrm{NMR}$ $\left(\delta, \mathrm{ppm}, \mathrm{DMSO}-d_{6}\right): 27.334,28.07,28.783,31.78$ (C4-8),
98.241 (C3), $115.266(\mathrm{CN}), 122.955\left(\mathrm{C3}^{\prime}, \mathrm{C}^{\prime}\right), 123.77\left(\mathrm{CF}_{3}\right)$, $125.577\left(\mathrm{C}^{\prime}\right), 126.274\left(\mathrm{C}^{\prime}, \mathrm{C}^{\prime}\right), 131.947(\mathrm{C} 3 \mathrm{a}), 135.634$ (C8a), $142.855\left(\mathrm{Cl}^{\prime}\right), 146.787(\mathrm{C} 2)$, and $176.507(\mathrm{C}=\mathrm{S})$; DART-TOF-MS $(\mathrm{m} / z)$ : $396.08[\mathrm{M}+\mathrm{H}]^{+}$; anal. calcd. for $\mathrm{C}_{18} \mathrm{H}_{16} \mathrm{~F}_{3} \mathrm{~N}_{3} \mathrm{~S}_{2}$ (395.07): C, 54.67; H, 4.08; F, 14.41; N, 10.63; and S, 16.21; found: C, 54.77; H, 3.99; F, 14.46; N, 10.68; and S, 16.16 .

2.2.4. General Synthetic Procedure for Compounds (7a-f). Few drops of sulfuric acid were added to a mixture of the appropriate lead compound $\mathbf{2 d - f}(5.6 \mathrm{mmol})$ and 1 equivalent of 4-(trifluoromethyl)benzaldehyde or 4-(2-pyridyl) benzaldehyde in absolute ethanol. Precipitation occurred once sulfuric acid was added. The precipitate was filtered, washed with absolute ethanol, and dried.

2-((4-(Trifluoromethyl)benzylidene)amino)-5,6-dihydro4H-cyclopenta[b] thiophene-3-carbonitrile (7a). Dark brown solid; yield: $41 \%$; mp: $150-152^{\circ} \mathrm{C}$; IR $\left(\mathrm{KBr}, v_{\max }, \mathrm{cm}^{-1}\right)$ : $2219.12(\mathrm{CN}), 1678.47(\mathrm{C}=\mathrm{N})$, and $1567.51,1536.64(\mathrm{C}=\mathrm{C})$; ${ }^{1} \mathrm{H}$ NMR $\left(\delta, \mathrm{ppm}, \mathrm{CDCl}_{3}\right): 2.444(m, 2 \mathrm{H}, \mathrm{H} 5), 2.873(t, 2 \mathrm{H}$, $\mathrm{H} 6), 2.946(t, 2 \mathrm{H}, \mathrm{H} 4), 7.704\left(d, 2 \mathrm{H}, J=7.8 \mathrm{~Hz}, \mathrm{H}^{\prime}, \mathrm{H}^{\prime}\right)$, $8.033\left(d, 2 \mathrm{H}, J=7.8 \mathrm{~Hz}, \mathrm{H} 2^{\prime}, \mathrm{H}^{\prime}\right)$, and $8.468(s, 1 \mathrm{H}, \mathrm{N}=\mathrm{CH})$; ${ }^{13} \mathrm{C}$ NMR $\left(\delta, \mathrm{ppm}, \mathrm{CDCl}_{3}\right): 27.379,28.169,30.239(\mathrm{C} 4-6)$, $103.913(\mathrm{C} 3), 114.492(\mathrm{CN}), 124.611\left(\mathrm{CF}_{3}\right), 125.814\left(\mathrm{C}^{\prime}\right.$, C5 $\left.{ }^{\prime}\right), 129.44\left(\mathrm{C}^{\prime}, \mathrm{C} 6^{\prime}\right), 133.441\left(\mathrm{C}^{\prime}\right), 138.087$ (C3a), $139.007\left(\mathrm{C}^{\prime}\right), 145.108$ (C6a), 155.649 (C2), and 163.744 $(\mathrm{N}=\mathrm{CH})$; and DART-TOF-MS $(\mathrm{m} / \mathrm{z}): 321.07[\mathrm{M}+\mathrm{H}]^{+}$; anal. calcd. for $\mathrm{C}_{16} \mathrm{H}_{11} \mathrm{~F}_{3} \mathrm{~N}_{2} \mathrm{~S}$ (320.06): C, 59.99; H, 3.46; F, 17.79; $\mathrm{N}, 8.75$; and $\mathrm{S}, 10.01$; found: C, 60.07; H, 3.39; F, 17.84; N, 8.80; and S, 9.97 .

2-((4-(Trifluoromethyl)benzylidene)amino)-4,5,6,7-tetrahydrobenzo[b]thiophene-3-carbonitrile ( $7 \boldsymbol{b})$. Yellow solid; yield: 78\%; mp: $146-147^{\circ} \mathrm{C}$; IR (KBr, $\left.v_{\max }, \mathrm{cm}^{-1}\right)$ : $2216.15(\mathrm{CN})$, $1659.53(\mathrm{C}=\mathrm{N})$, and $1563.09,1514.72(\mathrm{C}=\mathrm{C}) ;{ }^{1} \mathrm{H}$ NMR $(\delta$, ppm, $\left.\mathrm{CDCl}_{3}\right): 1.861(m, 4 \mathrm{H}, \mathrm{H} 5, \mathrm{H} 6), 2.664(s, 2 \mathrm{H}, \mathrm{H} 7), 2.711$ $(s, 2 \mathrm{H}, \mathrm{H} 4), 7.696\left(d, 2 \mathrm{H}, J=7.8 \mathrm{~Hz}, \mathrm{H}^{\prime}, \mathrm{H}^{\prime}\right), 8.026(d, 2 \mathrm{H}$, $\left.J=7.8 \mathrm{~Hz}, \mathrm{H}^{\prime}, \mathrm{H}^{\prime}\right)$, and $8.422(s, 1 \mathrm{H}, \mathrm{N}=\mathrm{CH}) ;{ }^{13} \mathrm{C} \mathrm{NMR}$ $\left(\delta, \mathrm{ppm}, \mathrm{CDCl}_{3}\right): 21.914,22.987,24.282,25.271$ (C4-7), 108.39 (C3), $114.201(\mathrm{CN}), 124.618\left(\mathrm{CF}_{3}\right), 125.799\left(\mathrm{C}^{\prime}\right.$, $\left.\mathrm{C}^{\prime}\right), 129.448\left(\mathrm{C}^{\prime}, \mathrm{C}^{\prime}\right), 133.426\left(\mathrm{C} 4^{\prime}\right), 133.832(\mathrm{C} 3 \mathrm{a})$, $135.572\left(\mathrm{C}^{\prime}\right), 138.102(\mathrm{C} 7 \mathrm{a}), 156.73(\mathrm{C} 2)$, and 158.569 $(\mathrm{N}=\mathrm{CH})$; and DART-TOF-MS $(\mathrm{m} / z): 335.09[\mathrm{M}+\mathrm{H}]^{+}$; anal. calcd. for $\mathrm{C}_{17} \mathrm{H}_{13} \mathrm{~F}_{3} \mathrm{~N}_{2} \mathrm{~S}$ (334.08): C, 61.07; H, 3.92; F, 17.05; $\mathrm{N}, 8.38$; and S, 9.59; found: C, 61.12; H, 3.86; F, 16.98; N, 8.42; and $S, 9.55$.

2-((4-(Trifluoromethyl)benzylidene)amino)-5,6,7,8-tetrahydro-4H-cyclohepta[b] thiophene-3-carbonitrile (7c). Yellow solid; yield: 99\%; $\mathrm{mp}$ : $113-114^{\circ} \mathrm{C}$; IR $\left(\mathrm{KBr}, v_{\max }, \mathrm{cm}^{-1}\right)$ : $2221.48(\mathrm{CN}), 1684.98(\mathrm{C}=\mathrm{N})$, and 1547.17, $1517.48(\mathrm{C}=\mathrm{C})$; ${ }^{1} \mathrm{H} \mathrm{NMR}\left(\delta, \mathrm{ppm}, \mathrm{CDCl}_{3}\right): 1.7(m, 4 \mathrm{H}, \mathrm{H} 5, \mathrm{H} 7), 1.882(s, 2 \mathrm{H}$, $\mathrm{H} 6), 2.804(t, 4 \mathrm{H}, \mathrm{H} 4, \mathrm{H} 8), 7.7\left(d, 2 \mathrm{H}, J=7.8 \mathrm{~Hz}, \mathrm{H}^{\prime}, \mathrm{H}^{\prime}\right)$, $8.026\left(d, 2 \mathrm{H}, J=7.8 \mathrm{~Hz}, \mathrm{H} 2^{\prime}, \mathrm{H}^{\prime}\right)$, and $8.438(s, 1 \mathrm{H}, \mathrm{N}=\mathrm{CH})$; ${ }^{13} \mathrm{C}$ NMR $\left(\delta, \mathrm{ppm}, \mathrm{CDCl}_{3}\right): 27.073,27.755,29.142,30.691$, $31.956(\mathrm{C} 4-8), 110.605(\mathrm{C} 3), 114.729(\mathrm{CN}), 122.824\left(\mathrm{CF}_{3}\right)$, $125.776\left(\mathrm{C} 3^{\prime}, \mathrm{C}^{\prime}\right), 129.356\left(\mathrm{C}^{\prime}, \mathrm{C} 6^{\prime}\right), 133.150\left(\mathrm{C}^{\prime}\right)$, 137.558 (C3a), $138.186\left(\mathrm{Cl}^{\prime}\right), 140.624$ (C8a), 156.538 $(\mathrm{C} 2)$, and $156.576(\mathrm{~N}=\mathrm{CH})$; and DART-TOF-MS $(\mathrm{m} / z)$ : $349.1[\mathrm{M}+\mathrm{H}]^{+}$; anal. calcd. for $\mathrm{C}_{18} \mathrm{H}_{15} \mathrm{~F}_{3} \mathrm{~N}_{2} \mathrm{~S}$ (348.09): C, 
62.06; H, 4.34; F, 16.36; N, 8.04; and S, 9.20; found: C, 62.12; $\mathrm{H}, 4.30 ; \mathrm{F}, 16.45 ; \mathrm{N}, 8.0$; and $\mathrm{S}, 9.25$.

2-((4-(Pyridin-2-yl)benzylidene)amino)-5,6-dihydro- $4 \mathrm{H}$ cyclopenta[b]thiophene-3-carbonitrile (7d). Yellow solid; yield: $29 \%$; mp: $191-193^{\circ} \mathrm{C}$; IR $\left(\mathrm{KBr}, v_{\max }, \mathrm{cm}^{-1}\right): 2219.27$ $(\mathrm{CN})$, and $1581.62,1548.56(\mathrm{C}=\mathrm{C}) ;{ }^{1} \mathrm{H}$ NMR $(\delta, \mathrm{ppm}$, $\left.\mathrm{CDCl}_{3}\right): 2.433(m, 2 \mathrm{H}, J=7.2 \mathrm{~Hz}, \mathrm{H} 5), 2.869(t, 2 \mathrm{H}$, $J=7.2 \mathrm{~Hz}, \mathrm{H} 6), 2.934(t, 2 \mathrm{H}, J=7.2 \mathrm{~Hz}, \mathrm{H} 4), 7.273(t, 1 \mathrm{H}$, $\left.\mathrm{H} 4^{\prime \prime}\right), 7.794\left(m, 2 \mathrm{H}, J=7.2 \mathrm{~Hz}, \mathrm{H} 5^{\prime \prime}, \mathrm{H} 6^{\prime \prime}\right), 8.032(d, 2 \mathrm{H}$, $\left.J=7.8 \mathrm{~Hz}, \mathrm{H}^{\prime}, \mathrm{H} 6^{\prime}\right), 8.106\left(d, 2 \mathrm{H}, J=7.8 \mathrm{~Hz}, \mathrm{H}^{\prime}, \mathrm{H}^{\prime}\right)$, $8.488(s, 1 \mathrm{H}, \mathrm{N}=\mathrm{CH})$, and $8.719\left(d, 1 \mathrm{H}, \mathrm{H} 33^{\prime \prime}\right) ;{ }^{13} \mathrm{C} \mathrm{NMR}$ ( $\left.\delta, \mathrm{ppm}, \mathrm{CDCl}_{3}\right): 27.356,28.215,30.223$ (C4-6), 102.779 (C3), $114.814(\mathrm{CN}), 120.946\left(\mathrm{C6}^{\prime \prime}\right), 122.794\left(\mathrm{C4}^{\prime \prime}\right), 127.294$, $129.869\left(\mathrm{C} 2^{\prime}-3^{\prime}, \mathrm{C}^{\prime}-6^{\prime}\right), 135.404(\mathrm{C} 3 \mathrm{a}), 136.906\left(\mathrm{Cl}^{\prime}\right)$, 137.887 (C5"), 142.809 (C6a), $144.787\left(\mathrm{C}^{\prime}\right), 149.884\left(\mathrm{C3}^{\prime \prime}\right)$, $156.185\left(\mathrm{Cl}^{\prime \prime}\right), 157.236(\mathrm{C} 2)$, and $164.986(\mathrm{~N}=\mathrm{CH})$; and DART-TOF-MS $(\mathrm{m} / z)$ : $330.11[\mathrm{M}+\mathrm{H}]^{+}$; anal. calcd. for $\mathrm{C}_{20} \mathrm{H}_{15} \mathrm{~N}_{3} \mathrm{~S}$ (329.10): C, 72.92; H, 4.59; N, 12.76; and S, 9.73; found: C, 72.88; H, 4.55; N, 12.81; and S, 9.69.

2-((4-(Pyridin-2-yl)benzylidene)amino)-4,5,6,7-tetrahydro benzo[b]thiophene-3-carbonitrile (7e). Yellow solid; yield: 83\%; mp: 191-193 ${ }^{\circ} \mathrm{C}$; IR (KBr, $\left.v_{\max }, \mathrm{cm}^{-1}\right)$ : $2214.28(\mathrm{CN})$, and $1582.55,1552.3(\mathrm{C}=\mathrm{C}) ;{ }^{1} \mathrm{H}$ NMR $\left(\delta, \mathrm{ppm}, \mathrm{CDCl}_{3}\right): 1.853$ ( $m, 4 \mathrm{H}, \mathrm{H} 5-6), 2.68(d, 4 \mathrm{H}, \mathrm{H} 4, \mathrm{H} 7), 7.272\left(m, 1 \mathrm{H}, \mathrm{H} 4{ }^{\prime \prime}\right)$, $7.788\left(s, 2 \mathrm{H}, \mathrm{H} 5^{\prime \prime}, \mathrm{H} 6^{\prime \prime}\right), 8.019\left(d, 2 \mathrm{H}, J=7.8 \mathrm{~Hz}, \mathrm{H} 2^{\prime}, \mathrm{H}^{\prime}\right)$, $8.094\left(d, 2 \mathrm{H}, J=7.8 \mathrm{~Hz}, \mathrm{H}^{\prime}, \mathrm{H}^{\prime}\right), 8.433(s, 1 \mathrm{H}, \mathrm{N}=\mathrm{CH})$, and $8.719\left(s, 1 \mathrm{H}, \mathrm{H} 33^{\prime \prime}\right) ;{ }^{13} \mathrm{C} \mathrm{NMR}\left(\delta, \mathrm{ppm}, \mathrm{CDCl}_{3}\right): 21.984,23.05$, 24.299, 25.25 (C4-5), 107.256 (C3), 114.538 (CN), 120.969 (C6"), $122.817\left(\mathrm{C}^{\prime \prime}\right), 127.278\left(\mathrm{C3}^{\prime}, \mathrm{C}^{\prime}\right), 129.869\left(\mathrm{C}^{\prime}, \mathrm{C}^{\prime}\right)$, 132.797 (C3a), $135.235\left(\mathrm{Cl}^{\prime}\right), 135.434\left(\mathrm{C5}^{\prime \prime}\right), 136.96(\mathrm{C} 7 \mathrm{a})$, 142.739 (C4'), 149.853 (C3"), 156.147 (C1"), 158.27 (C2), and $159.749(\mathrm{~N}=\mathrm{CH})$; and DART-TOF-MS $(\mathrm{m} / \mathrm{z}): 344.12$ $[\mathrm{M}+\mathrm{H}]^{+}$; anal. calcd. for $\mathrm{C}_{21} \mathrm{H}_{17} \mathrm{~N}_{3} \mathrm{~S}$ (343.11): C, 73.44; $\mathrm{H}$, 4.99; N, 12.24; and S, 9.33; found: C, 73.48; H, 4.94; N, 12.31; and $S, 9.27$.

2-((4-(Pyridin-2-yl)benzylidene)amino)-5,6,7,8tetrahydro-4H-cyclohepta [b]thiophene-3-carbonitrile (7f). Yellow solid; yield: $86 \%$; mp: $199-200^{\circ} \mathrm{C}$; IR $\left(\mathrm{KBr}, v_{\max }\right.$, $\left.\mathrm{cm}^{-1}\right): 2218.75(\mathrm{CN})$, and 1582.43, $1546.89(\mathrm{C}=\mathrm{C}) ;{ }^{1} \mathrm{H}$ $\operatorname{NMR}\left(\delta, \mathrm{ppm}, \mathrm{CDCl}_{3}\right): 1.703(m, 4 \mathrm{H}, \mathrm{H} 5, \mathrm{H} 7), 1.88(s, 2 \mathrm{H}$, H6), $2.801(m, 4 \mathrm{H}, \mathrm{H} 4, \mathrm{H} 8), 7.275\left(s, 1 \mathrm{H}, \mathrm{H} 44^{\prime \prime}\right), 7.795(m$, $\left.2 \mathrm{H}, \mathrm{H} 5^{\prime \prime}, \mathrm{H} 6^{\prime \prime}\right), 8.026\left(d, 2 \mathrm{H}, J=7.8 \mathrm{~Hz}, \mathrm{H} 2^{\prime}, \mathrm{H} 6^{\prime}\right), 8.102$ $\left(d, 2 \mathrm{H}, J=7.8 \mathrm{~Hz}, \mathrm{H}^{\prime}, \mathrm{H}^{\prime}\right), 8.463(s, 1 \mathrm{H}, \mathrm{N}=\mathrm{CH})$, and 8.722 $\left(s, 1 \mathrm{H}, \mathrm{H} 3{ }^{\prime \prime}\right) ;{ }^{13} \mathrm{C}$ NMR $\left(\delta, \mathrm{ppm}, \mathrm{CDCl}_{3}\right): 27.126,27.809$, $29.15,30.653,31.994(\mathrm{C} 4-8), 109.532(\mathrm{C} 3), 115.036(\mathrm{CN})$, 120.946 (C6"), 122.771 (C4"), $127.286\left(\mathrm{C3}^{\prime}, \mathrm{C5}^{\prime}\right), 129.777$ $\left(\mathrm{C} 2^{\prime}, \mathrm{C}^{\prime}\right), 135.519(\mathrm{C} 3 \mathrm{a}), 136.462\left(\mathrm{C1}^{\prime}\right), 136.922\left(\mathrm{C}^{\prime \prime}\right)$, 140.279 (C8a), $142.701\left(\mathrm{C4}^{\prime}\right), 149.861$ (C3"), $156.224\left(\mathrm{Cl}^{\prime \prime}\right)$, $157.726(\mathrm{C} 2)$, and $158.155(\mathrm{~N}=\mathrm{CH})$; and DART-TOF-MS $(\mathrm{m} / z)$ : $358.14[\mathrm{M}+\mathrm{H}]^{+}$; anal. calcd. for $\mathrm{C}_{22} \mathrm{H}_{19} \mathrm{~N}_{3} \mathrm{~S}$ (357.13): C, 73.92; H, 5.36; N, 11.75; and S, 8.97; found: C, 73.97; H, 5.29; N, 11.81; and S, 9.02 .

2.3. X-Ray Crystallographic Analysis. Compounds $\mathbf{4 c}$ and $7 \mathbf{e}$ were obtained as single crystals by slow evaporation of the ethanol solution of the pure compounds at room temperature. Crystallographic data were collected using a Bruker APEX-II D8 Venture area diffractometer, equipped with graphite monochromatic Mo K $\alpha$ radiation, $\lambda=0.71073$, and $\mathrm{Cu} \mathrm{K} \alpha$ radiation, $\lambda=1.54178 \AA$ at 293 (2) K. Cell refinement and data reduction were carried out using Bruker SAINT. SheLXT was used to determine the structure [24, 25]. The final refinement was carried out by the full-matrix leastsquares technique with anisotropic thermal data for -nonhydrogen atoms on F-CCDC 1823351 and 1534088 that contain the supplementary crystallographic data for these compounds obtained free of charge from the Cambridge Crystallographic Data Centre (http://www.ccdc.cam.ac.uk/data request/cif).

\subsection{Biology}

2.4.1. Evaluation of Antimicrobial Activity. All selected organisms (0.5 McFarland standards) were thoroughly mixed with sterilized Mueller-Hinton agar (MHA). This suspension $(25 \mathrm{~mL})$ was placed into Petri dishes $(90 \mathrm{~mm}$ diameter) and left to cool and solidify by placing the Petri dishes on a cool horizontal surface. A $10 \mathrm{~mm}$ diameter well was holed on both sides of the agar plate by using a sterilized hollow cylinder as a template. The formulations and control antibiotics $(1 \mathrm{mg} / \mathrm{mL})$ were placed into each well $(50 \mu \mathrm{L})$ to permit diffusion. All plates were incubated at $37 \pm 0.5^{\circ} \mathrm{C}$ for $24 \mathrm{~h}$ in aerobic conditions; the test was performed in triplicate. The antimicrobial activities of the selected formulations and control antibiotics against the tested microorganisms were compared. The diameter of the inhibition zone was measured with a gauge and expressed in $\mathrm{mm}$ (mean \pm standard deviation (SD)).

2.4.2. Evaluation of Anti-Inflammatory Activity. Fresh whole human blood was collected and mixed with equal volumes of sterilized Alsever's solution (2\% dextrose, $0.8 \%$ sodium citrate, $0.05 \%$ citric acid, $0.42 \%$ sodium chloride, and $100 \mathrm{~mL}$ of distilled water). This blood solution was centrifuged at $3,000 \mathrm{rpm}$ for $10 \mathrm{~min}$ and then washed three times with an equal volume of normal saline. The volume of blood was measured, and it was reconstituted with normal saline to prepare $10 \% v / v$ suspension. The reaction mixture consisted of $1 \mathrm{~mL}$ of the test sample in normal saline at different concentrations: $0.5 \mathrm{~mL}$ of $10 \%$ human red blood cell (HRBC) suspension, $1 \mathrm{~mL}$ of $0.2 \mathrm{M}$ phosphate buffer, and $1 \mathrm{~mL}$ of hypotonic saline. They were incubated at $37^{\circ} \mathrm{C}$ for $30 \mathrm{~min}$ and centrifuged at $3,000 \mathrm{rpm}$ for $30 \mathrm{~min}$. Hemoglobin content in the supernatant was determined spectrophotometrically at $560 \mathrm{~nm}$. Each experiment was performed in triplicate. Diclofenac sodium was used as a standard, and distilled water was used as a control. The blood control represented $100 \%$ lysis or zero percent stability.

\section{Results and Discussion}

3.1. Chemistry. Using the Gewald method for thiophene synthesis, we synthesized the lead compounds (2a-f) (Scheme 1) [26, 27]. 


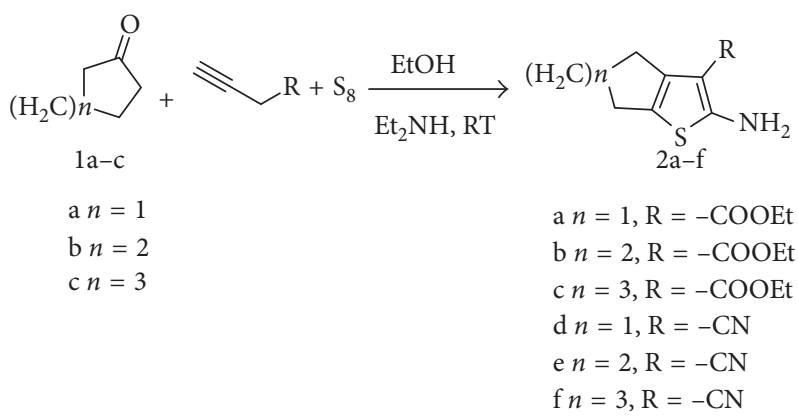

Scheme 1: Gewald thiophene synthesis.
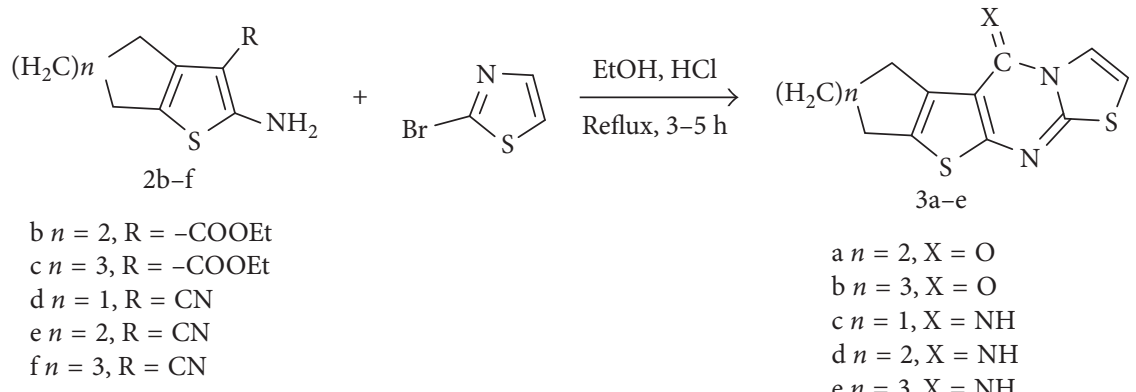

$$
\begin{aligned}
& \text { a } n=2, \mathrm{X}=\mathrm{O} \\
& \text { b } n=3, \mathrm{X}=\mathrm{O} \\
& \text { c } n=1, \mathrm{X}=\mathrm{NH} \\
& \mathrm{d} n=2, \mathrm{X}=\mathrm{NH} \\
& \text { e } n=3, \mathrm{X}=\mathrm{NH}
\end{aligned}
$$

Scheme 2: Synthesis of thieno[2,3-(d)] thiazolo[3,2-(a)]pyrimidine derivatives (3a-e).

A mixture of the appropriate compound (2b-f), 2bromothiazole, and few drops of hydrochloric acid in ethanol was refluxed for $3-5 \mathrm{~h}$ to produce thieno[2,3-d] thiazolo $[3,2-a]$ pyrimidine derivatives (Scheme 2).

This reaction involves nucleophilic substitution in bromothiazole followed by nucleophilic substitution on the ester group to form the pyrimidinone ring (compounds $\mathbf{3 a - b}$ ). However, for compounds $\mathbf{3 c}-\mathbf{e}$, nucleophilic substitution was followed by nucleophilic addition of a cyanide group to the thiazole nitrogen to form the pyrimidinimine ring.

IR, ${ }^{1} \mathrm{H}$ NMR, ${ }^{13} \mathrm{C} \mathrm{NMR}$, and mass spectral data of the polycyclic compound $\mathbf{3 a}$ and new compounds $\mathbf{3 b}-\mathbf{e}$ were consistent with the assigned structures. The IR spectrum of compound $\mathbf{3 b}$ showed an absorption band at $1674.41 \mathrm{~cm}^{-1}$ for the carbonyl group, whereas there was no amino group absorption band. The ${ }^{1} \mathrm{H}$ NMR spectrum showed the presence of the cycloheptenyl protons and appearance of new signals: two doublet signals at $\delta=6.855$ and $7.984 \mathrm{ppm}$ (each integrates for one proton corresponding to the two protons of the thiazole ring) with coupling constant $(J)=$ $4.8 \mathrm{~Hz}$, whereas there was no signal for the ethyl group linked to the ester group. In ${ }^{13} \mathrm{C} N \mathrm{NMR}$, the three carbons of the thiazole group appeared at $\delta=109.494,121.667$, and $156.982 \mathrm{ppm}$, carbonyl peak appeared at $\delta=162.317 \mathrm{ppm}$, and no peaks for the ethyl group. Finally, the direct analysis in real-time/time-of-flight mass spectrometry (DART-TOFMS) spectrum showed a molecular ion peak $[\mathrm{M}+\mathrm{H}]^{+}$at $m / z=277.05$.

The lead compounds (2b-f) were refluxed in bromoalkanoyl chloride derivatives (Scheme 3 ) to produce compounds $(\mathbf{4 a - e})$ by nucleophilic substitution reaction.
However, the nucleophilic substitution reaction between compound $\mathbf{2 e}$ and 6-bromohexanoyl chloride was followed by nucleophilic addition of a cyanide group to the carbonyl oxygen to form the oxazinimine ring of the novel compound 5 (Scheme 4).

The IR data of compound 4d showed the appearance of an $\mathrm{NH}$ absorption band at $3360.95 \mathrm{~cm}^{-1}$ and another absorption band with two heads at $1675.80 \mathrm{~cm}^{-1}$, which represents the two carbonyl groups. In ${ }^{1} \mathrm{H}$ NMR, five new signals appeared: multiplet at $\delta=1.508,1.756$, and $1.888 \mathrm{ppm}$ and triplet at $\delta=2.456$ and $3.396 \mathrm{ppm}$ (each signal integrates for two protons corresponding to the protons of the side chain). In addition, one proton singlet signal for $\mathrm{NH}$ appeared at $\delta=11.2 \mathrm{ppm}$. The ${ }^{13} \mathrm{C}$ NMR spectrum showed the appearance of the five carbons of the side chain in the aliphatic range; besides, it showed signals for the cycloheptenyl carbons in the range of $\delta=24.442-36.564 \mathrm{ppm}$ and a peak for the amide group at $\delta=166.748 \mathrm{ppm}$. Finally, the DART-TOFMS spectrum showed a molecular ion peak $[\mathrm{M}+\mathrm{H}]^{+}$at $m / z=416.09$.

For compound 5, the disappearance of the cyanide absorption band in the IR spectrum, appearance of one proton signal for $\mathrm{NH}$ at $\delta=12.177 \mathrm{ppm}$ in the ${ }^{1} \mathrm{H} \mathrm{NMR}$ spectrum, and appearance of carbon peaks at $\delta=159.013$ and $163.559 \mathrm{ppm}$ for $\mathrm{C} 4$ and $\mathrm{C} 2$, respectively, in the ${ }^{13} \mathrm{C}$ NMR spectrum proved the formation of the oxazinimine ring. The six protons of the side chain appeared in the ${ }^{1} \mathrm{H}$ NMR spectrum as multiplet signals at $\delta=1.376$ and 1.641-1.816 ppm for $\mathrm{H} 2-\mathrm{H} 4^{\prime}$ and two triplet signals at $\delta=2.533$ and $3.508 \mathrm{ppm}$ for $\mathrm{H}^{\prime}$ and $\mathrm{H}^{\prime}$, respectively (each signal integrates for two protons). Moreover, the

${ }^{13} \mathrm{C}$ NMR spectrum showed the five carbons of the side 


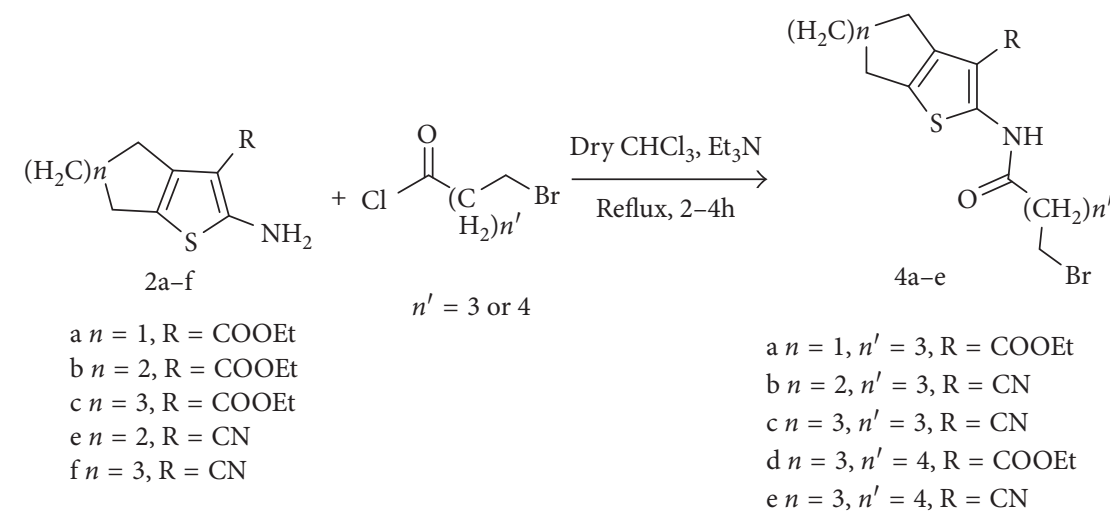

Scheme 3: Synthesis of compounds $\mathbf{4 a - e . ~}$

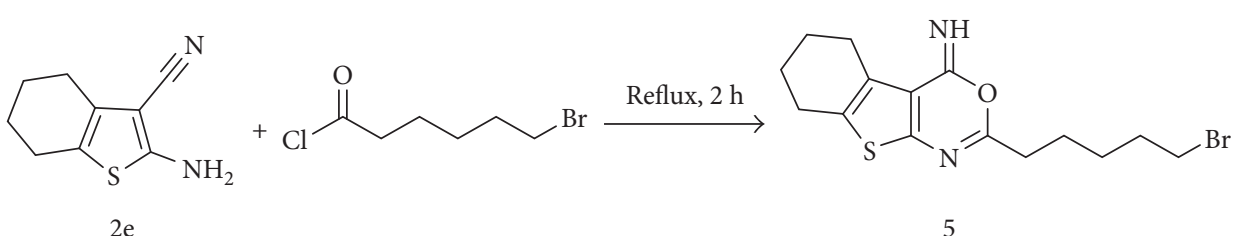

Scheme 4: Synthesis of thieno[2,3-(d)][1,3] oxazin-4-imine derivative (5).

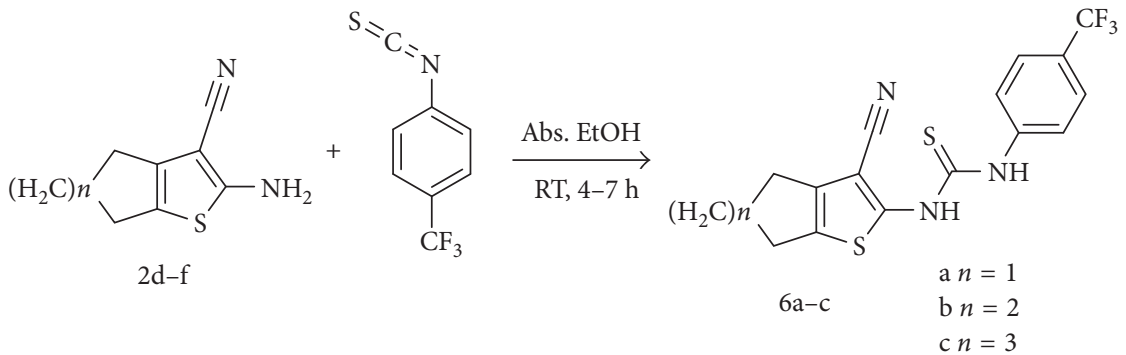

Scheme 5: Synthesis of thiophene-thiourea derivatives $(\mathbf{6 a}-\mathbf{c})$.

chain in the aliphatic range. Finally, the molecular weight was confirmed by the appearance of a molecular ion peak $[\mathrm{M}+\mathrm{H}]^{+}$at $m / z=355.05$ in the DART-TOF-MS spectrum.

A mixture of the appropriate lead compound (2d-f) and 4-(trifluoromethyl phenyl) isothiocyanate in absolute ethanol was stirred at $25^{\circ} \mathrm{C}$ for $2-7 \mathrm{~h}$. The new thiophenethiourea derivatives $(\mathbf{6 a}-\mathbf{c})$ were obtained by nucleophilic addition (Scheme 5).

Structures of compounds $(\mathbf{6 a}-\mathbf{c})$ were confirmed by the presence of a cyanide group and appearance of (4-(trifluoromethyl)phenyl)thiourea bands and peaks in the IR, ${ }^{1} \mathrm{H}$ NMR, and ${ }^{13} \mathrm{C}$ NMR spectra. Herein, for compound $\mathbf{6 b}$, the IR spectrum showed two absorption bands for the two $\mathrm{NH}$ groups at 3328.78 and $3235.86 \mathrm{~cm}^{-1}$ and an absorption band for cyanide at $2210.19 \mathrm{~cm}^{-1}$. The ${ }^{1} \mathrm{H}$ NMR spectrum showed a doublet signal $(2 \mathrm{H})$ with $J=7.8 \mathrm{~Hz}$ at $\delta=7.715 \mathrm{ppm}$ for $\mathrm{H} 2^{\prime}$ and $\mathrm{H}^{\prime}$, doublet signal $(2 \mathrm{H})$ with $J=7.8 \mathrm{~Hz}$ at $\delta=7.838 \mathrm{ppm}$ for $\mathrm{H}^{\prime}{ }^{\prime}$ and $\mathrm{H}^{\prime}$, and two singlet signals for $\mathrm{NH}$ at $\delta=10.765$ and $11.016 \mathrm{ppm}$ (each integrates for one proton). The ${ }^{13} \mathrm{C}$ NMR spectrum showed a peak for cyanide at $\delta=114.791 \mathrm{ppm}$; additionally, the phenyl ring carbons appeared as follows: $\mathrm{C}^{\prime}$ and $\mathrm{C}^{\prime}$ at $\delta=123.07 \mathrm{ppm}, \mathrm{C} 2^{\prime}$ and $\mathrm{C}^{\prime}{ }^{\prime}$ at $\delta=126.297 \mathrm{ppm}, \mathrm{C} 4^{\prime}$ at $\delta=130.988 \mathrm{ppm}^{\prime}$, and $\mathrm{C}^{\prime}{ }^{\prime}$ at $\delta=142.862 \mathrm{ppm} . \mathrm{CF}_{3}$ appeared at $\delta=123.77 \mathrm{ppm}$, whereas the thionyl group appeared at $\delta=176.728 \mathrm{ppm}$. Finally, the molecular weight was confirmed by the appearance of a molecular ion peak $[\mathrm{M}+\mathrm{H}]^{+}$at $m / z=382.07$ in the DARTTOF-MS spectrum.

Addition of few drops of sulfuric acid to a mixture of the appropriate lead compound (2d-f) and 4-(trifluoromethyl) benzaldehyde or 4-(2-pyridyl) benzaldehyde in absolute ethanol resulted in instant precipitation of the product (Scheme 6).

All structures of the novel synthesized compounds $(7 \mathbf{a}-\mathbf{f})$ were confirmed by the existence of a cyanide group, disappearance of $\mathrm{NH}_{2}$, and appearance of 4-(trifluoromethyl) benzylidene or 4-(pyridin-2-yl)benzylidene bands and peaks in the IR, ${ }^{1} \mathrm{H}$ NMR, and ${ }^{13} \mathrm{C}$ NMR spectra. For instance, compound $\mathbf{7 d}$ showed a cyanide absorption band at 


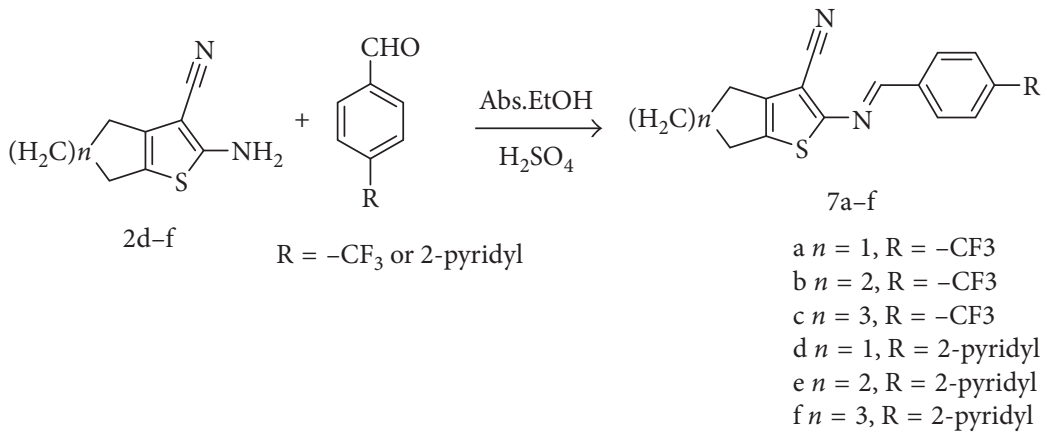

Scheme 6: Synthesis of thiophene-3-carbonitrile derivatives $(\mathbf{7 a - f})$.

TABLE 1: Experimental detail of the crystalline structures of $\mathbf{4 c}$ and $\mathbf{7 e}$.

Crystal data
Chemical formula
Molecular weight
Crystal system, space group
Temperature (K)
$a, b, c(\AA)$
$\alpha, \beta, \gamma\left(^{\circ}\right)$
$V\left(\AA^{3}\right)$
$Z$
Radiation type
$\mu($ mm
Crystal size (mm)
Data collection
Diffractometer
Absorption correction
$T_{\text {min }}, T_{\text {max }}$
Number of measured, independent,
and observed $[I>2 \sigma(I)]$ reflections
$R_{\text {int }}$
Refinement
$R\left[F^{2}>2 \sigma\left(F^{2}\right)\right]$, wR $\left(F^{2}\right), S$
Number of reflections
Number of parameters
Number of restraints
$\Delta \rho_{\text {max }}, \Delta \rho_{\text {min }}\left(\mathrm{e} \AA^{-3}\right)$
CCDC number

$4 \mathrm{c}$
$\mathrm{C}_{15} \mathrm{H}_{19} \mathrm{BrN}_{2} \mathrm{OS}$
355.29
Triclinic, $P-1$
293
$7), 9.0388(13), 15.825(2)$
$5), 97.557(5), 90.726(5)$
$805.76(18)$
2
Mo Ka
2.68
$0.55 \times 0.42 \times 0.04$

Bruker APEX-II D8 venture diffractometer Multiscan SADABS Bruker 2014 $0.893,0.921$

16032,3700 , and 1871

0.152

$0.114,0.341,1.66$

3700

176

0

$1.37,-1.74$

1823351

\author{
7 e \\ $\mathrm{C}_{21} \mathrm{H}_{17} \mathrm{~N}_{3} \mathrm{~S}$ \\ 343.44 \\ Triclinic, $P-1$ \\ 293 \\ $868.13(13)$ \\ 2 \\ Mo Ka \\ 0.19 \\ $0.4 \times 0.29 \times 0.1$
}

6.6424 (6), 11.5386 (10), 11.5868 (10)

98.088 (3), 93.063 (3), 98.051 (3)

Bruker APEX-II D8 venture diffractometer Multiscan SADABS Bruker 2014 $0.927,0.982$

33436,3412 , and 2377

0.103

$0.066,0.201,1.08$

3412

226

1

$0.34,-0.33$

1534088
$2219.27 \mathrm{~cm}^{-1}$ and disappearance of the $\mathrm{NH}_{2}$ bands in the IR spectrum. The ${ }^{1} \mathrm{H}$ NMR spectrum showed signals of the pyridyl ring protons as follows: a triplet signal $(1 \mathrm{H})$ at $\delta=7.273 \mathrm{ppm}$ for $\mathrm{H} 4^{\prime \prime}$, multiplet signal $(2 \mathrm{H})$ at $\delta=7.794 \mathrm{ppm}$ for $\mathrm{H}^{\prime \prime}$ and $\mathrm{H}^{\prime \prime}$, and doublet signal at $\delta=8.719 \mathrm{ppm}$ for $\mathrm{H}^{\prime \prime}$. In addition, the four protons of the benzene ring appeared as two doublet signals (each integrates for two protons) with $J=7.2 \mathrm{~Hz}$ at $\delta=8.032$ and $8.106 \mathrm{ppm}$. Moreover, an $\mathrm{N}=\mathrm{CH}$ singlet signal $(1 \mathrm{H})$ appeared at $\delta=8.488 \mathrm{ppm} .{ }^{13} \mathrm{C}$ NMR showed a cyanide peak $(\delta=$ $114.814 \mathrm{ppm}$ ), pyridyl ring peaks (C6" at $\delta=120.946 \mathrm{ppm}$, $\mathrm{C}^{\prime \prime}$ peak at $\delta=122.794 \mathrm{ppm}, \mathrm{C}^{\prime \prime}$ peak at $\delta=137.887 \mathrm{ppm}$, C3" peak at $\delta=149.884 \mathrm{ppm}$, and $\mathrm{C}^{\prime \prime}$ peak at $\delta=$ $156.185 \mathrm{ppm})$, benzene ring peaks $\left(\mathrm{C} 2^{\prime}\right.$ and $\mathrm{C} 3^{\prime}$ at $\delta=127.294$, $\mathrm{C}^{\prime}$ and $\mathrm{C}^{\prime}$ at $129.869 \mathrm{ppm}^{\prime} \mathrm{C}^{\prime}$ at $\delta=136.906 \mathrm{ppm}$, and $\mathrm{C}^{\prime}$ at $\delta=144.787 \mathrm{ppm}$ ), and $\mathrm{N}=\mathrm{CH}$ peak at $\delta=164.986 \mathrm{ppm}$. DART-TOF-MS confirmed the molecular weight of the expected structure, as evidenced by the appearance of a molecular ion peak $[\mathrm{M}+\mathrm{H}]^{+}$at $m / z=330.11$.

3.2. X-Ray Crystallography. The structures of three of the synthesized compounds (4c, 7e) were examined by X-ray crystallography. The crystallographic data and refinement information are summarized in Table 1. As shown in Figure 2, the asymmetric units contained one independent molecule.

\subsection{Biology}

3.3.1. Evaluation of Antimicrobial Activity. An in vitro antimicrobial study was performed using the agar diffusion method to evaluate the ability of the synthesized compounds to inhibit microbial growth, as previously described by 

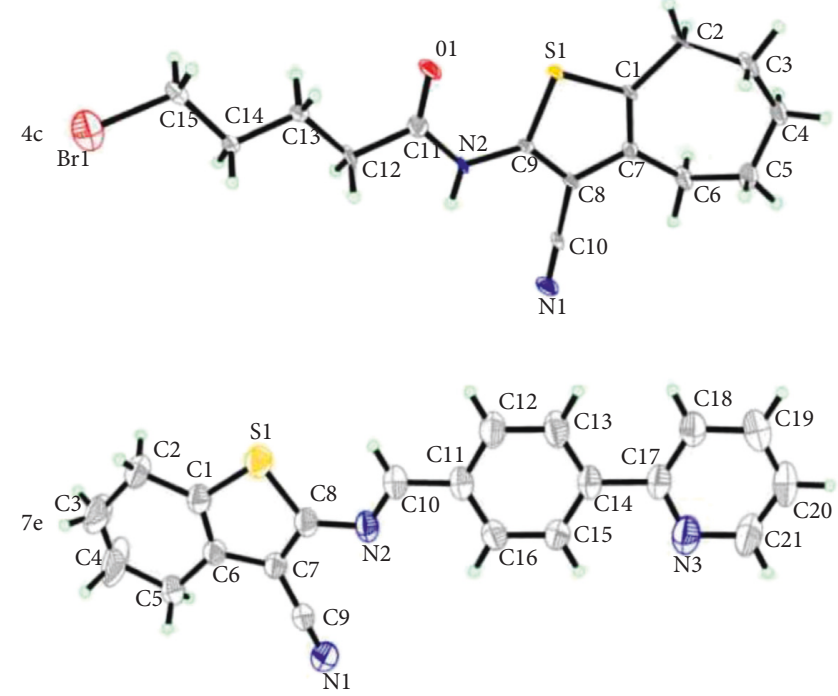

Figure 2: ORTEP diagrams of the titled compounds $4 \mathbf{c}$ and $7 \mathbf{e}$. Displacement ellipsoids are plotted at the $40 \%$ probability level for non-H atoms.

TABle 2: The zones of inhibition ( $\mathrm{mm}$ ) of the synthesized compounds and standard antimicrobial drugs.

\begin{tabular}{|c|c|c|c|c|c|c|c|}
\hline \multirow{2}{*}{ Compound number } & \multicolumn{7}{|c|}{ Zone of inhibition (mm) } \\
\hline & Staphylococcus aureus & Bacillus & Escherichia coli & Klebsiella & Salmonella & Pseudomonas & Candida \\
\hline $3 \mathbf{a}$ & $11 \pm 0.186$ & - & - & - & - & - & - \\
\hline $3 \mathbf{b}$ & - & - & - & - & - & - & - \\
\hline $3 c$ & - & - & - & - & - & - & - \\
\hline $3 e$ & - & - & - & - & - & - & - \\
\hline $5 \mathbf{b}$ & - & - & - & - & - & - & - \\
\hline 5 & $16 \pm 0.136$ & $13 \pm 0.501$ & - & $12 \pm 0.42$ & $12 \pm 0.075$ & - & - \\
\hline $4 b$ & - & - & - & - & - & $14 \pm 0.056$ & - \\
\hline $4 c$ & - & - & - & - & - & $10 \pm 0.138$ & - \\
\hline $6 a$ & $15 \pm 0.322$ & $15 \pm 0.894$ & - & - & - & - & - \\
\hline $6 b$ & $10 \pm 0.27$ & - & - & - & - & - & - \\
\hline $6 c$ & $13 \pm 0.273$ & $12 \pm 0.071$ & - & - & - & - & - \\
\hline Ampicillin & $30 \pm 0.057$ & $15 \pm 0.069$ & $25 \pm 0.07$ & $13 \pm 0.062$ & $34 \pm 0.059$ & $18 \pm 0.054$ & - \\
\hline Fluconazole & - & - & - & - & - & - & $38 \pm 0.037$ \\
\hline
\end{tabular}

Bonev et al. [28]. The antimicrobial activities of the synthesized compounds (3a-c, $\mathbf{3 e}, \mathbf{4 e}, \mathbf{4 b - c , 5}$, and $\mathbf{6 a - c})$ were tested against some selected microorganisms, including Staphylococcus aureus, Bacillus species, Escherichia coli, Klebsiella species, Salmonella species, Pseudomonas species, and Candida albicans (Table 2).

As shown in Table 2, results of the antimicrobial activity studies revealed that the test compounds displayed variable inhibitory effects against the growth of the tested bacteria. Interestingly, compound $\mathbf{6 a}$ was equally potent to ampicillin against Bacillus species. The antimicrobial potencies of other derivatives, particularly compound $\mathbf{5}$ (against Klebsiella and Bacillus), were potentially comparable to those of ampicillin. The antifungal activity study revealed that the tested compounds exhibited no activity against Candida albicans.

3.3.2. Evaluation of Anti-Inflammatory Activity. Compounds $3 \mathbf{a}-\mathbf{c}, 3 \mathbf{e}, 4 \mathbf{a}-\mathbf{e}, \mathbf{5}, \mathbf{6} \mathbf{a}-\mathbf{c}$, and $7 \mathbf{a}-\mathbf{f}$ were screened in vitro for anti-inflammatory activity, using a method previously described by Mahajan et al. [29]. The anti-inflammatory activity of the thiazole and thiophene derivatives (Table 3) showed that compounds $\mathbf{3 e}, \mathbf{4} \mathbf{b}$, and $\mathbf{6 a}$ possessed more potent anti-inflammatory activity than that of diclofenac sodium. Compounds $\mathbf{4 d}$ and $\mathbf{7 b}$ showed good antiinflammatory activity, whereas compounds $\mathbf{5}, \mathbf{7 d}$, and $\mathbf{7 f}$ showed moderate activity.

\section{Conclusions}

In this study, we successfully synthesized some thiazole, pyrimidine, and thiophene derivatives. Among them, fifteen new target compounds were prepared. In addition, seven compounds (4c, 7e) were successfully obtained as pure crystals. Antimicrobial activity studies revealed that the test compounds displayed broad antibacterial spectrum and good potency. Interestingly, compound $\mathbf{6 a}$ was equally potent to ampicillin against Bacillus strains. According to the 
TABLE 3: In vitro anti-inflammatory activity of the synthesized compounds and standard drug (diclofenac sodium).

\begin{tabular}{lcc}
\hline $\begin{array}{l}\text { Compound } \\
\text { number }\end{array}$ & $\begin{array}{c}\text { Spectrophotometer } \\
+\mathrm{RBC}\end{array}$ & $\begin{array}{c}\text { Spectrophotometer }+\mathrm{RBC} \\
(\% \text { hemolysis })\end{array}$ \\
\hline $\mathbf{3 a}$ & $0.011 \pm 0.0068$ & $101.13 \pm 0.0031$ \\
$\mathbf{3 b}$ & $0.013 \pm 0.000516$ & $102.1542 \pm 0.021$ \\
$\mathbf{3 c}$ & $0.009 \pm 0.000473$ & $102.6048 \pm 0.00402$ \\
$\mathbf{3 e}$ & $0.179 \pm 0.01775$ & $101.6216 \pm 0.0005$ \\
$\mathbf{4 d}$ & $0.011 \pm 0.0068$ & $101.13 \pm 0.0031$ \\
$\mathbf{4 e}$ & $0.098 \pm 0.001227$ & $102.686 \pm 0.00211$ \\
$\mathbf{5}$ & $0.101 \pm 0.01862$ & $101.9084 \pm 0.0002$ \\
$\mathbf{4 a}$ & $0.017 \pm 0.0075$ & $101.908 \pm 0.002404$ \\
$\mathbf{4 d}$ & $0.219 \pm 0.0225$ & $102.727 \pm 0.0126$ \\
$\mathbf{4 c}$ & $0.032 \pm 0.002$ & $102.441 \pm 0.01308$ \\
$\mathbf{6 a}$ & $0.146 \pm 0.001366$ & $102.523 \pm 0.0187$ \\
$\mathbf{6 b}$ & $0.01 \pm 0.00493$ & $102.154 \pm 0.00312$ \\
$\mathbf{6 c}$ & $0.046 \pm 0.001789$ & $102.236 \pm 0.0107$ \\
7a & $0.023 \pm 0.000894$ & $102.0723 \pm 0.02135$ \\
7b & $0.113 \pm 0.001033$ & $102.318 \pm 0.0042$ \\
7c & $0.029 \pm 0.003225$ & $102.113 \pm 0.028$ \\
7d & $0.066 \pm 0.00324$ & $102.2 \pm 0.00301$ \\
7e & $0.052 \pm 0.0041$ & $102.154 \pm 0.0014$ \\
7f & $0.06 \pm 0.00319$ & $101.908 \pm 0.01405$ \\
Diclofenac & $0.132 \pm 0.002251$ & $102.851 \pm 0.00076$ \\
sodium & $0.261 \pm 0.002683$ & - \\
Control & $0.428 \pm 0.002442$ & - \\
Blank & & \\
\hline & & -
\end{tabular}

anti-inflammatory activity test, compounds $\mathbf{3 e}, \mathbf{4 b}$, and $\mathbf{6 a}$ possessed greater anti-inflammatory potency than that of the standard drug. Therefore, compound 6a was shown to exhibit both antibacterial and anti-inflammatory activities, which could be beneficial in the treatment of various diseases.

\section{Data Availability}

The data used to support the findings of this study are available from the corresponding author upon request.

\section{Conflicts of Interest}

The authors declare that they have no conflicts of interest.

\section{Acknowledgments}

The authors thank the Deanship of Scientific Research and RSSU at King Saud University for their technical support.

\section{References}

[1] L. J. Lombardo, F. Y. Lee, P. Chen et al., "Discovery of $N$-(2Chloro-6-methyl- phenyl)-2-(6-(4-(2-hydroxyethyl)- piperazin1-yl)-2-methylpyrimidin-4- ylamino)thiazole-5-carboxamide (BMS-354825), a dual Src/Abl kinase inhibitor with potent antitumor activity in preclinical assays," Journal of Medicinal Chemistry, vol. 47, no. 27, pp. 6658-6661, 2004.

[2] J. Das, P. Chen, D. Norris et al., "2-Aminothiazole as a novel kinase inhibitor template. Structure-activity relationship studies toward the discovery of $\mathrm{N}$-(2-Chloro-6methylphenyl)-2-[[6-[4-(2-hydroxyethyl)-1-piperazinyl)]-2methyl-4-pyrimidinyl]amino)]-1,3-thiazole-5-carboxamide (dasatinib, BMS-354825) as a potent pan-Src kinase inhibitor," Journal of Medicinal Chemistry, vol. 49, no. 23, pp. 6819-6832, 2006.

[3] B. L. Mylari, E. R. Larson, T. A. Beyer et al., "Novel, potent aldose reductase inhibitors: 3,4-dihydro-4-oxo-3-[[5-(trifluoromethyl)2-benzothiazolyl]methyl]-1-phthalazineacetic acid (zopolrestat) and congeners," Journal of Medicinal Chemistry, vol. 34, no. 1, pp. 108-122, 1991.

[4] M. C. Van Zandt, M. L. Jones, D. E. Gunn et al., "Discovery of 3[(4,5,7-trifluorobenzothiazol-2-yl)methyl]indole- $N$-acetic acid (lidorestat) and congeners as highly potent and selective inhibitors of aldose reductase for treatment of chronic diabetic complications," Journal of Medicinal Chemistry, vol. 48, no. 9, pp. 3141-3152, 2005.

[5] B. Ettinger, D. M. Black, B. H. Mitlak et al., "Reduction of vertebral fracture risk in postmenopausal women with osteoporosis treated with raloxifene: results from a 3-year randomized clinical trial. multiple outcomes of raloxifene evaluation (MORE) investigators," JAMA, vol. 282, no. 7, pp. 637-645, 1999.

[6] S. R. Cummings, S. Eckert, K. A. Krueger et al., "The effect of raloxifene on risk of breast cancer in postmenopausal women: results from the MORE randomized trial. Multiple outcomes of raloxifene evaluation," JAMA, vol. 281, no. 23, pp. 21892197, 1999.

[7] V. G. Vogel, J. P. Costantino, D. L. Wickerham et al., "Effects of tamoxifen vs raloxifene on the risk of developing invasive breast cancer and other disease outcomes: the NSABP study of tamoxifen and raloxifene (STAR) P-2 trial," JAMA, vol. 295, no. 23 , pp. $2727-2741,2006$.

[8] V. G. Vogel, J. P. Costantino, D. L. Wickerham et al., "Update of the National Surgical Adjuvant Breast and Bowel Project Study of Tamoxifen and Raloxifene (STAR) P-2 Trial: preventing breast cancer," Cancer Prevention Research, vol. 3, no. 6, pp. 696-706, 2010.

[9] J. M. Herbert, D. Frehel, E. Vallee et al., "A novel antithrombotic agent," Cardiovascular Drug Reviews, vol. 11, no. 2, pp. 180-198, 1993.

[10] J. M. Herbert, A. Tissinier, G. Defreyn, and J. P. Maffrand, "Inhibitory effect of clopidogrel on platelet adhesion and intimal proliferation after arterial injury in rabbits," Arteriosclerosis, Thrombosis, and Vascular Biology, vol. 13, no. 8, pp. 1171-1179, 1993.

[11] M. Gent, "A randomised, blinded, trial of clopidogrel versus aspirin in patients at risk of ischaemic events (CAPRIE). CAPRIE Steering Committee," The Lancet, vol. 348, no. 9038, pp. 1329-1339, 1996.

[12] K. Starčević, G. Karminski-Zamola, I. Piantanida, M. Zinić, L. Sǔman, and M. Kralj, "Photo-induced switch of a DNA/ RNA inactive molecule into a classical intercalator," Journal of the American Chemical Society, vol. 127, no. 4, pp. 1074-1075, 2005.

[13] R. Romagnoli, P. G. Baraldi, M. D. Carrion et al., "Design, synthesis and biological evaluation of thiophene analogues of chalcones," Bioorganic and Medicinal Chemistry, vol. 16, no. 10, pp. 5367-5376, 2008.

[14] I. C. F. R. Ferreira, M. R. P. Queiroz, M. Vilas-Boas, L. M. Estevinho, A. Begouin, and G. Kirsch, "Evaluation of the antioxidant properties of diarylamines in benzo[b]thiophene series by free radical scavenging activity and reducing power," 
Bioorganic and Medicinal Chemistry Letters, vol. 16, no. 5, pp. 1384-1387, 2006.

[15] I. L. Pinto, R. L. Jarvest, and H. T. Serafinowska, "The synthesis of 5-alkoxy and 5-amino substituted thiophenes," Tetrahedron Letters, vol. 41, pp. 1597-1600, 2000.

[16] P. G. Baraldi, M. G. Pavani, J. C. Shryock et al., "Synthesis of 2-amino-3-heteroaroylthiophenes and evaluation of their activity as potential allosteric enhancers at the human A1 receptor," European Journal of Medicinal Chemistry, vol. 39, no. 10, pp. 855-865, 2004.

[17] A. D. Pillai, P. D. Rathod, F. P. Xavier, K. K. Vasu, H. Padh, and V. Sudarsanam, "Design, synthesis, and pharmacological evaluation of some 2-[4-morpholino]-3-aryl-5-substituted thiophenes as novel anti-inflammatory agents: generation of a novel anti-inflammatory pharmacophore," Bioorganic and Medicinal Chemistry, vol. 12, no. 17, pp. 4667-4671, 2004.

[18] F. M. Moghaddam and H. Zali-Boinee, "A versatile one-pot synthesis of 2,3,5-tri-substituted thiophenes from thiomorpholides," Tetrahedron Letters, vol. 44, no. 33, pp. 62536255, 2003.

[19] V. H. Rawal, R. J. Jones, and M. P. Cava, "Photocyclization strategy for the synthesis of antitumor agent CC-1065: synthesis of dideoxy PDE-I and PDE-II. Synthesis of thiophene and furan analogs of dideoxy PDE-I and PDE-II," Journal of Organic Chemistry, vol. 52, no. 1, pp. 19-28, 1987.

[20] K. Starčević, M. Kralj, I. Piantanida, L. Šuman, K. Pavelić, and G. Karminski-Zamola, "Synthesis, photochemical synthesis, DNA binding and antitumor evaluation of novel cyano- and amidinosubstituted derivatives of naphtho-furans, naphthothiophenes, thieno-benzofurans, benzodithiophenes and their acyclic precursors," European Journal of Medicinal Chemistry, vol. 41, no. 8, pp. 925-939, 2006.

[21] H. Z. Shams, Y. M. Elkholy, R. A. Azzam, and R. M. Mohareb, "Synthetic potentialities of thiophene systems in heterocyclic synthesis: a novel synthesis of thieno[2,3-b]pyridine derivatives," Phosphorus, Sulfur, and Silicon and the Related Elements, vol. 155, no. 1, pp. 215-233, 1999.

[22] H. Z. Shams, R. M. Mohareb, M. H. Helal, and A. E. Mahmoud, "Synthesis, structure elucidation, and biological evaluation of some fused and/or pendant thiophene, pyrazole, imidazole, thiazole, triazole, triazine, and coumarin systems based on cyanoacetic 2-[(benzoylamino)thioxomethyl]-hydrazide," Phosphorus, Sulfur, and Silicon and the Related Elements, vol. 182, no. 2, pp. 237-263, 2007.

[23] S. M. Sherif, R. M. Mohareb, H. Z. Shams, and H. M. Gaber, "A convenient synthesis of polyfunctionally substituted benzo [b]thiophen-2-yl-pyrimidine, -pyrazole, -isoxazole and pyridazine derivatives," Journal of Chemical Research, no. 11, pp. 434-435, 1995.

[24] G. M. Sheldrick, "A short history of SHELX," Acta Crystallographica Section A Foundations of Crystallography, vol. 64, no. 1, pp. 112-122, 2008.

[25] G. M. Sheldrick, SHELXTL-PC (Version 5.1), Siemens Analytical Instruments, Inc., Madison, WI, USA, 1997.

[26] K. Gewald, "Zur reaktion von a-oxo-mercaptanen mit nitrilen," Angewandte Chemie, vol. 73, no. 3, pp. 114-114, 1961.

[27] K. Gewald, E. Schinke, and H. Böttcher, "Heterocycles from $\mathrm{CH}$-acidic nitriles VII 2-aminothiophene, carbonyl compounds, and sulfur," Chemische Berichte, vol. 99, no. 1, pp. 94-100, 1966.

[28] B. Bonev, J. Hooper, and J. Parisot, "Principles of assessing bacterial susceptibility to antibiotics using the agar diffusion method," Journal of Antimicrobial Chemotherapy, vol. 61, no. 6, pp. 1295-1301, 2008.

[29] P. Mahajan, M. Nikam, A. Chate et al., "Synthesis, and antitubercular activity of new benzo[b]thiophenes," Phosphorus, Sulfur, and Silicon and the Related Elements, vol. 190, no. 11, pp. 1803-1813, 2015. 

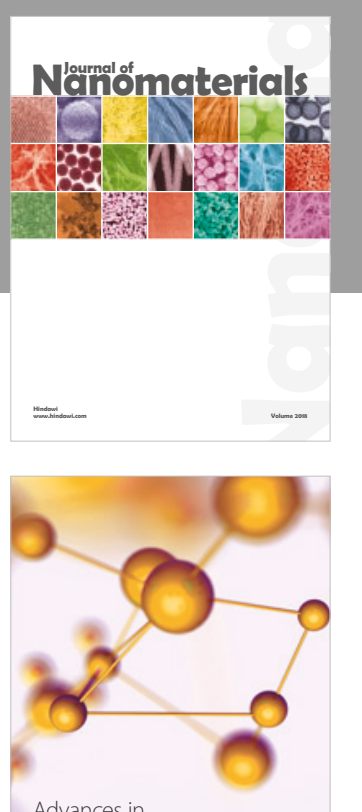

Physical Chemistry
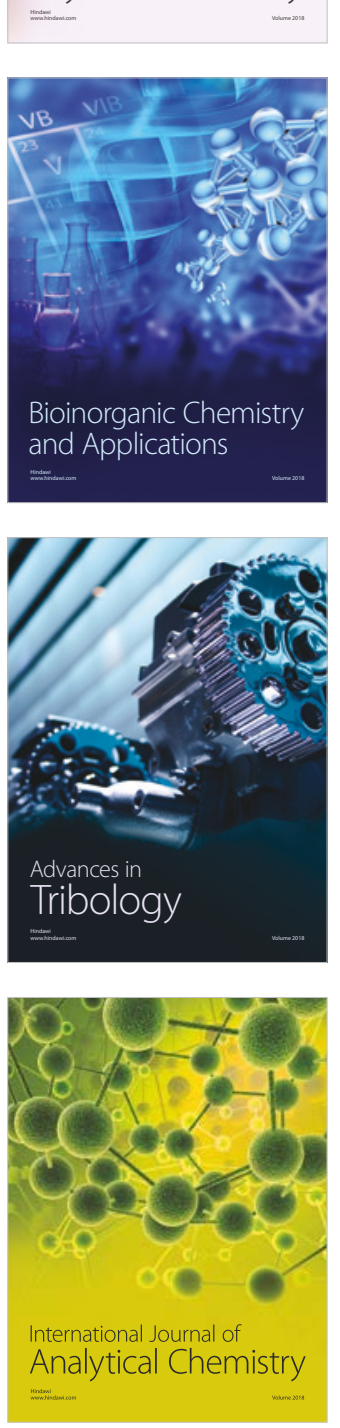

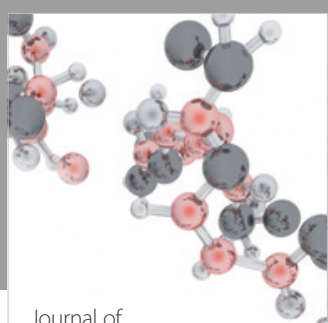

Analytical Methods

in Chemistry

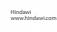

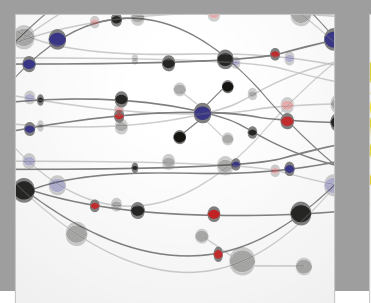

The Scientific World Journal

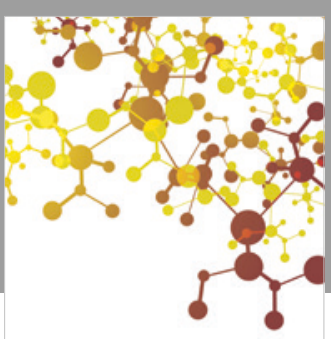

Journal of

Applied Chemistry
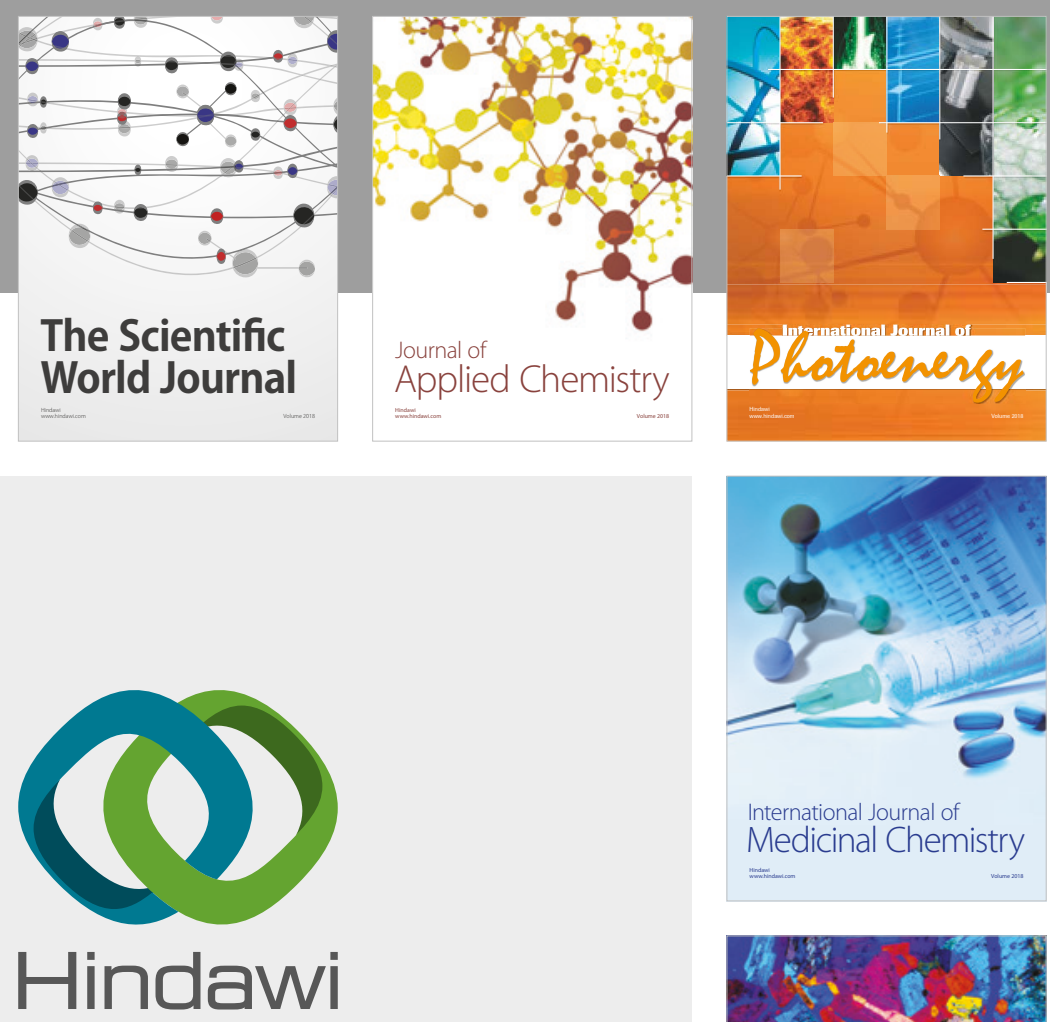

Submit your manuscripts at

www.hindawi.com
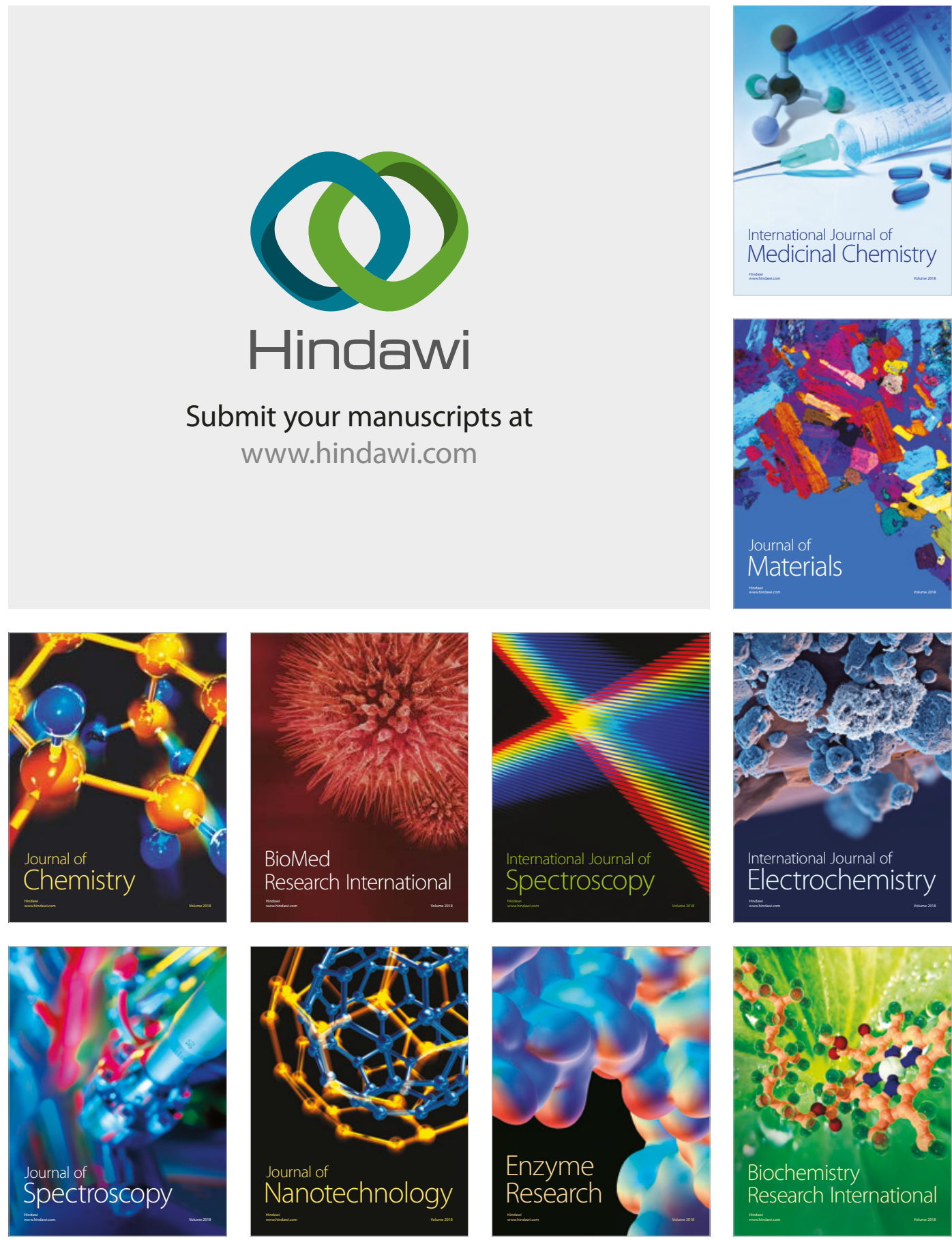
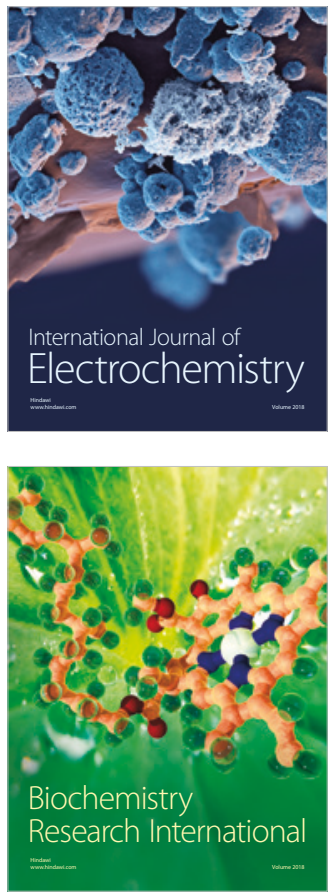